\title{
Comparison of Large Eddy Simulations against measurements from the Lillgrund offshore wind farm
}

\author{
Ishaan Sood ${ }^{1}$, Elliot Simon ${ }^{2}$, Athanasios Vitsas ${ }^{1}$, Bart Blockmans ${ }^{1}$, Gunner C. Larsen ${ }^{2}$, and \\ Johan Meyers ${ }^{1}$ \\ ${ }^{1}$ Mechanical Engineering, KU Leuven, Celestijnenlaan 300, Leuven 3001, Belgium \\ ${ }^{2}$ DTU Wind Energy, Technical University of Denmark, Lyngby 2800, Denmark
}

Correspondence: Ishaan Sood (ishaan.sood@kuleuven.be)

\begin{abstract}
Numerical simulation tools such as Large Eddy Simulations (LES) have been extensively used in recent years to simulate and analyze turbine-wake interactions within large wind farms. However, to ensure the reliability of the performance and accuracy of such numerical solvers, validation against field measurements is essential. To this end, a measurement campaign is carried out at the Lillgrund offshore wind farm to gather data for the validation of an in-house LES solver. Flow field data

5 is collected from the farm using three long-range WindScanners, along with turbine performance and load measurements from individual turbines. Turbulent inflow conditions are reconstructed from an existing precursor database using a scaling-andshifting approach, proposed so that the generated inflow statistics match the measurements. Thus, 5 different simulation cases are setup, corresponding to 5 different inflow conditions at the Lillgrund wind farm. Operation of the 48 Siemens $2.3 \mathrm{MW}$ turbines from the Lillgrund wind farm is parameterized in the flow domain using an Aeroelastic Actuator Sector Model (AASM).

10 Time-series turbine performance metrics from the simulated cases are compared against field measurements to evaluate the accuracy of the optimization framework, turbine model and flow solver. In general, results from the numerical solver show good comparison in terms of power production, turbine loading and wake recovery. Nevertheless, larger errors for a few turbines in the wind farm across the simulated cases reveal the need for an improved controller implementation, and possibly a finer simulation grid for capturing wake turbulence.
\end{abstract}

\section{Introduction}

Recent years have seen the emergence of wind-farm simulation tools that cover the whole chain from flow-coupled aeroelastic models to power-grid models. The complexity of these models ranges from analytical tools, which simplify wake expansion and merging, to complex Computational Fluid Dynamics (CFD) solvers which represent the turbines and their influence on the surrounding flow field. Amongst all these numerical tools, Large Eddy Simulations (LES) feature detailed representation of the turbulent flow in and around large wind farms (Munters and Meyers, 2018; Lin and Porté-Agel, 2019). This increased detail in simulating the physics governing wind-farm flows has facilitated the study of wind-farm aerodynamics and enabled the analysis of phenomena like turbine-wake interactions, gusts, atmospheric stratification and the effect of wind farms on local wind climate (Mehta et al., 2014). Additionally, LES has also been used to investigate and develop coordinated wind-farm control 
strategies, which could provide the benefits of power maximization, asset life extension and grid frequency regulation, thus improving the performance and capabilities of wind farms. (Goit and Meyers, 2015; Y1lmaz and Meyers, 2018; Bossanyi, 2018; Boersma et al., 2019; Frederik et al., 2020). However, to give credibility to these studies it is essential to validate numerical solvers against reliable measurement data. While wind-tunnel experiments provide a useful avenue for testing, their accuracy in representing full-scale wind farms is limited due to the size and measurement constraints of wind tunnels (Bastankhah and Porté-Agel, 2017). Therefore, proper validation of wind-farm numerical models requires accurate reference data in the form of detailed flow field and performance measurements from existing wind farms. To this end, a measurement campaign was carried out at the Lillgrund wind farm, located $10 \mathrm{~km}$ off the coast of southern Sweden, as part of the Horizon 2020 TotalControl project. The measurement campaign made use of 3 long-range LiDARs, which measure the inflow conditions for the farm while also resolving the flow field in a part of the Lillgrund wind farm for wake measurements. The flow field data was supplemented by simultaneous power and structural-load measurements from individual wind turbines. The combination of LiDAR inflow field data, turbine performance data, wake data and loading data provide a unique data set for the validation of coupled flow and aeroelastic solvers.

In this work, SP-Wind, an in-house aeroelastic LES solver, which has previously been used extensively for wind-farm modelling and control optimization, is used to simulate the operation of the Lillgrund wind farm during the measurement campaign. While previous wind-farm validation studies have been carried out using LES (Wu and Porté-Agel, 2011, 2013; Nilsson et al., 2014; Wu and Porté-Agel, 2015; Draper et al., 2016; Simisiroglou et al., 2018), this work differs on three fronts. First, the atmospheric conditions at the Lillgrund site are recreated in the numerical domain by analyzing incoming flow field LiDAR measurements. Second, instead of initializing the flow field from scratch, as is the convention for LES precursor simulations (Stevens et al., 2014), the current work proposes a framework for reusing a previously generated precursor flow database for matching the conditions during the measurement campaign, substantially reducing the associated computational costs and time for LES wind-farm validation studies. Third, the current study utilizes a novel Aeroelastic Actuator Sector Model (AASM) for parameterizing the turbine forces in the numerical domain (Vitsas and Meyers, 2016) . Compared to other actuator models such as the Actuator Disc Model (ADM) and the Actuator Line Model (ALM), the AASM has the advantage of accurately representing rotating turbine blades while allowing for coarser time steps through spatial and temporal filtering, and decoupling of the LES time step and the time step of the flexible multibody model that is a part of the AASM.

50 The present article is organized as follows: Section 2 details the specifics of the measurement campaign and available datasets. Section 3 presents the specifications of the numerical solver used in this study, while Section 4 outlines the optimization framework developed to recreate the atmospheric conditions at Lillgrund using a previously generated precursor data-set. A comparison of individual turbine performance results from the numerical solver against field measurements, and a wake deficit analysis are presented in Section 5. Finally, Section 6 outlines a summary of the validation and the challenges associated with LES validation studies of wind farms. 


\section{Lillgrund offshore measurement campaign}

\subsection{Lillgrund offshore wind farm}

The Lillgrund offshore wind farm is located approximately $10 \mathrm{~km}$ off the coast of southern Sweden, just south of the Öresund Bridge, where average wind speeds are overall close to $8.5 \mathrm{~m} \mathrm{~s}^{-1}$ (Sebastiani et al., 2021). The wind farm contains 48 wind turbines (Siemens SWT-2.3-93) with a total capacity of 110 megawatts (MW). The farm's turbines have a rotor diameter of 93 metres, hub height of 65 metres, and a tip height of 115 metres. The farm is known to suffer from performance losses, as the turbines originally intended for the farm were replaced by larger models, leading to a tighter layout when normalized by turbine diameter (Simisiroglou et al., 2018).

\subsection{LiDAR measurements}

65 During the measurement campaign from September 2019 to February 2020, three long-range WindScanners, i.e. pulsed scanning Doppler wind LiDARs (Vasiljević et al., 2016), were installed on the Lillgrund wind turbine transition pieces and used to measure the flow field both upstream and within the farm, in the layout shown in Figure 1.

\subsubsection{Inflow LiDAR}

The inflow measuring system on turbine B08, "Vara", performed repeating Plan Position Indicator (PPI) sector-scans with a constant elevation angle of $8^{\circ}$ and azimuth sweep of $60^{\circ}$. The center-line of the arc scan was intended to lie parallel with the B-row of turbines, however no hard targets were visible from its install location to allow for a precise alignment. Instead, the system was coarsely aligned during installation, and subsequently, the static misalignment was determined and later corrected for using the turbine's calibrated nacelle direction signal.

The inflow LiDAR data was processed firstly by removing periods of low signal quality, i.e periods with Carrier to Noise Ratio (CNR) below $-26 \mathrm{~dB}$. Partial scans with a low proportion $(<80 \%)$ of valid radial speed values were also filtered out. The remaining valid data was reconstructed into two-component horizontal wind vectors using the integrating Velocity-Azimuth Process (iVAP) method (Liang, 2007). Lastly, the direction misalignment due to imperfect installation of the system was corrected by determining the static offset between the turbine nacelle direction measurements and LiDAR reconstructed wind direction. The static direction misalignment was found to be $-20.47^{\circ}$.

A time-series of processed wind speed and direction inflow measurements corresponding to the turbines' hub height (65 m) is shown in Figure 2, with the equivalent wind rose shown in Figure 3. This location corresponds to the LiDAR range gate at a distance of 430 meters, given the LiDAR's inclined beam. Range gates between 70 and 1490 meters were sampled in steps of 20 meters. 
https://doi.org/10.5194/wes-2021-153

Preprint. Discussion started: 20 January 2022

(c) Author(s) 2022. CC BY 4.0 License.

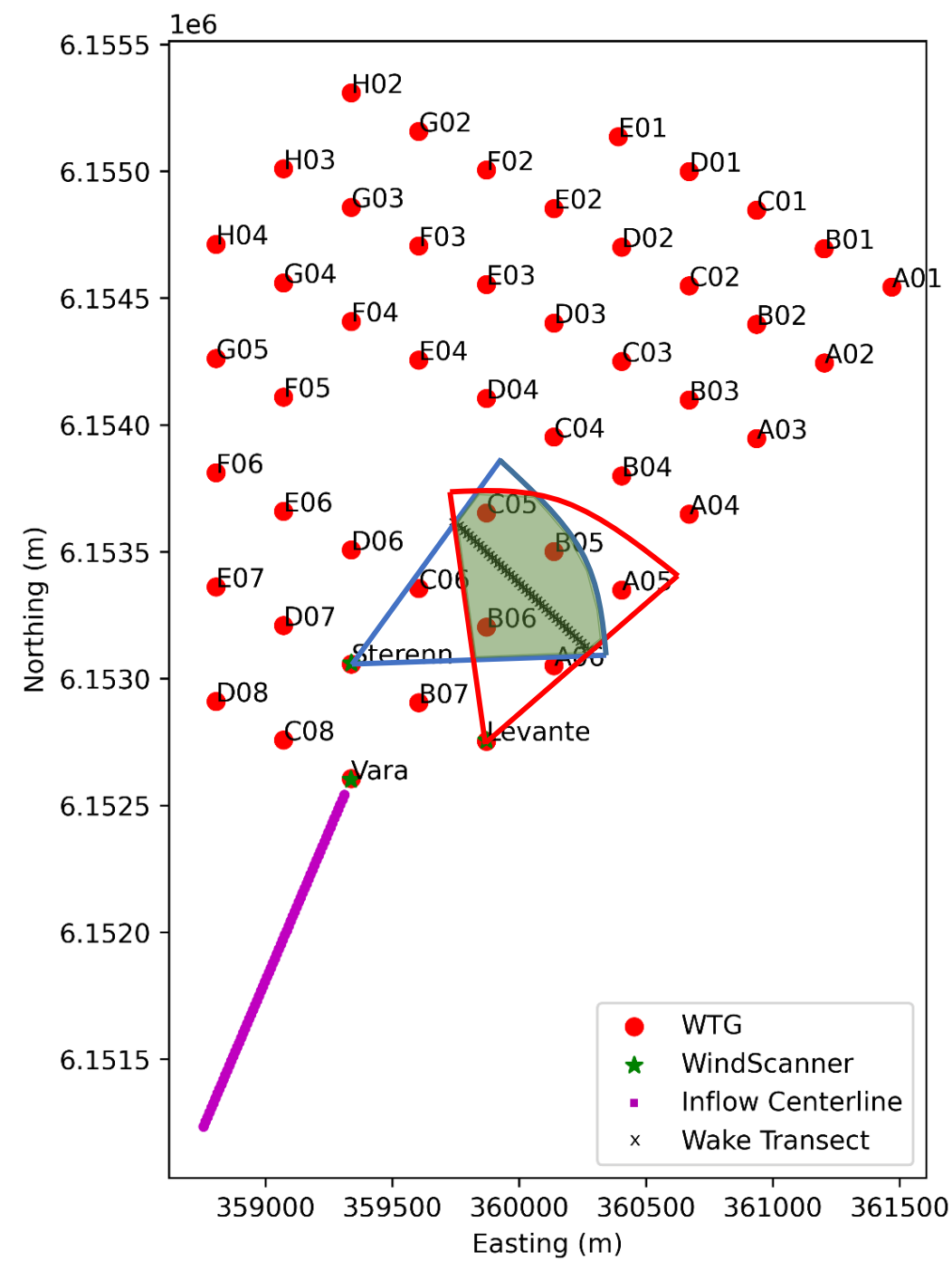

Figure 1. Top-down view of position and scan area for inflow and wake measuring LiDARs installed on turbines B08, A07 and C07. The time-space synchronized transect lines are marked with black X markings. The overlapping area shaded in green which lies off the transect is resolved by time-averaging. 

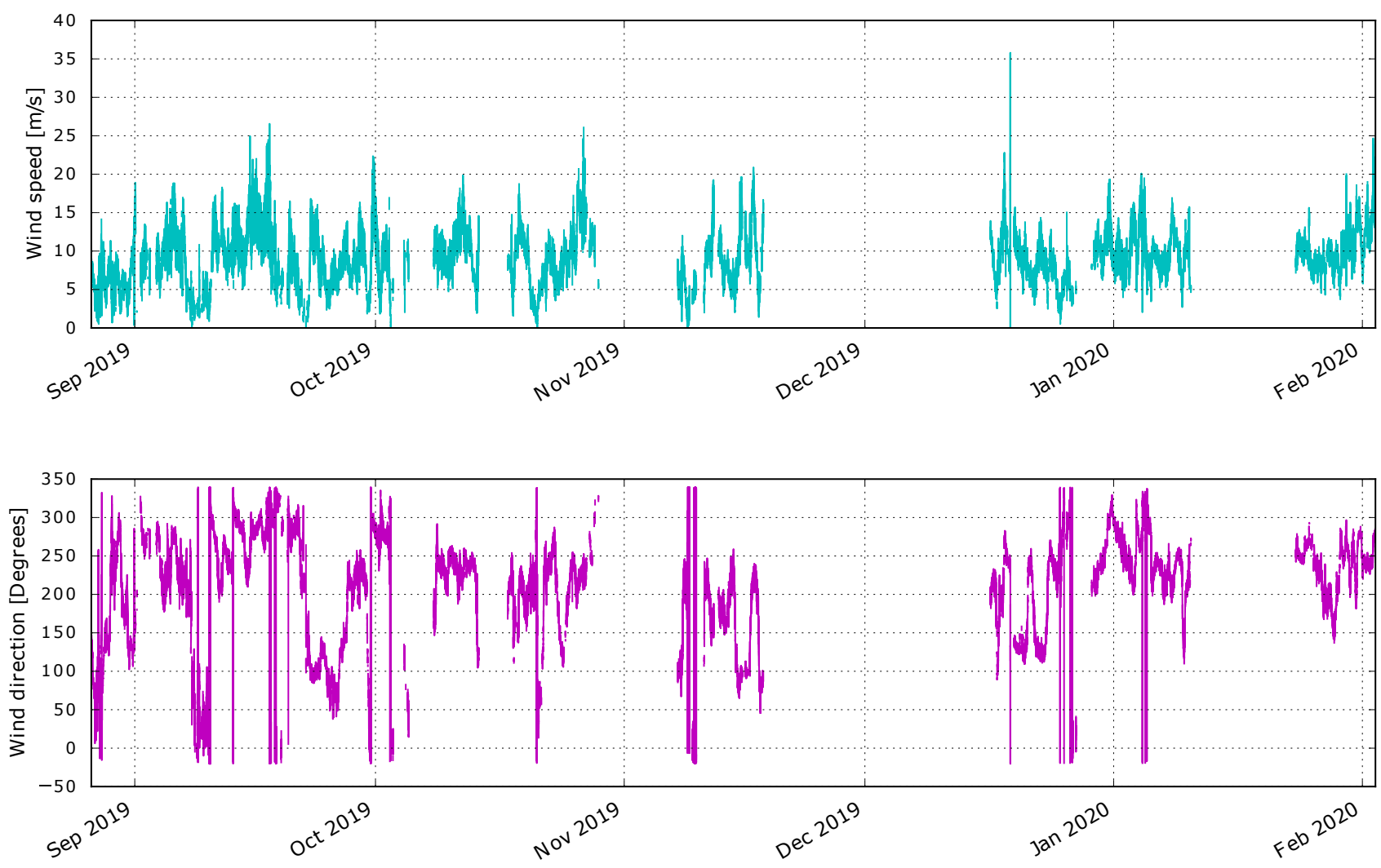

Figure 2. Wind-speed (top) and direction (bottom) measurements at hub height of $65 \mathrm{~m}$ processed from the inflow LiDAR mounted on turbine B08. Missing data corresponds to equipment downtime and filtering of low signal quality periods.

\subsubsection{Wake Scanning LiDARs}

85 Two additional scanning LiDARs identical to the inflow measuring system were installed within the wind farm to measure wake effects and intra-farm flows. The two wake LiDARs ("Levante" on A07 and "Sterenn" on C07) performed coordinated dualDoppler complex trajectory scans within the area shown in Figure 1. The intersecting measurement positions along the three transects were time and space synchronized using the DTU WindScanner software. Overlapping scan areas which lie away from the three transect lines are not time-space synchronized but have been averaged over a 10-minute period to produce a 3D wind field. From this, a horizontal slice has been taken to obtain the 2D wind field at constant hub height. The dual-Doppler wind retrieval method used in this study follows the process outlined in Simon and Courtney (2016). 


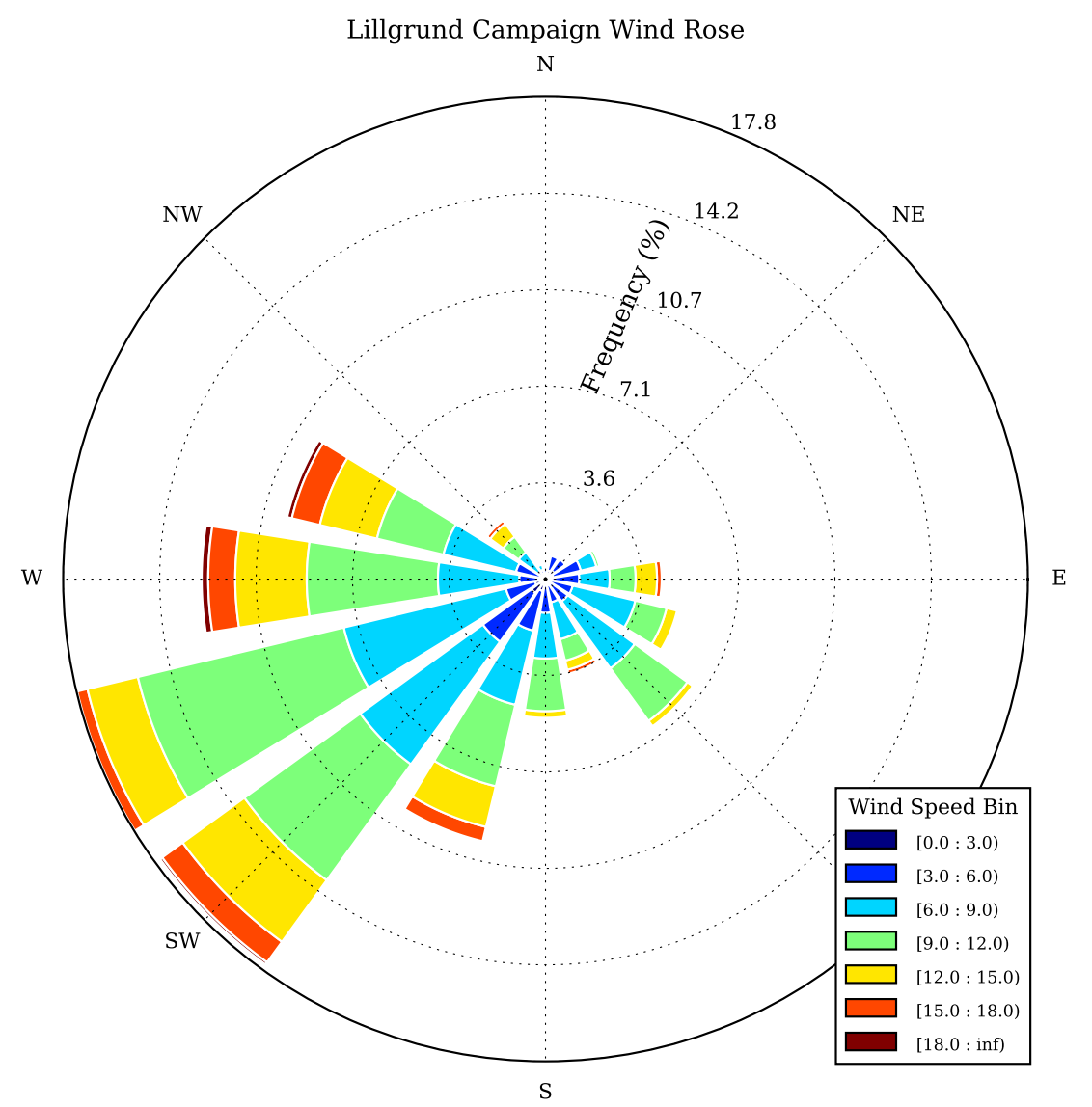

Figure 3. Wind rose depicting hub height wind speed and direction from the inflow measuring LiDAR.

\subsection{SCADA data}

Data from the wind farm monitoring system was provided by Vattenfall. This included the following channels from all 48 turbines in the wind farm: Active Power, Blade Angles (A/B/C), Nacelle Direction, Rotor RPM, and Wind Speed. The raw streaming data format did not maintain a specific time resolution, where deviations in the sampling rate existed between channels and over time. The data-set was processed into a constant sampling rate of $0.5 \mathrm{~Hz}$ timesteps across all channels. The raw nacelle direction signals were found to have differing offsets, and were then calibrated on a per-turbine basis and corrected in the final data-set. Given the fact that met mast data were not available, the calibration was performed using sets of turbines each consisting of of a front turbine and the corresponding closest downstream turbine. The inflow direction generating the largest wake loss at the downstream turbine location corresponds to the direction from the front turbine to the downstream turbine, whereby the offset of the measured nacelle direction at the front turbine follows directly. To cover the complete wind 
rose, 4 direction sectors of $90 \mathrm{deg}$. was defined, and within each of these the nacelle direction calibration was performed using $2-3$ sets of turbines.

\subsection{Load data}

Six wind turbines (designations: B06, B07, B08, C08, D07 and D08) have been outfitted in the past with load measuring equipment, and could be used during the TotalControl measurement campaign. These data are available starting November 2019 at $10 \mathrm{~Hz}$ sampling frequency. Strain gauge measurements are available both at the blade root (installed 1.5 meters from blade root) and tower base (installed 8.52 meters from tower base). The tower base position includes two sensors oriented 90 degrees apart. The sensors were installed and calibrated by Siemens Gamesa.

\section{Numerical solver}

\subsection{Fluid solver}

SP-Wind is a wind-farm Large Eddy Simulation code built on a high-order flow solver developed over the last 15 years at KU Leuven (Calaf et al., 2010; Allaerts and Meyers, 2015; Munters and Meyers, 2018). The three-dimensional, unsteady, and spatially filtered Navier-Stokes momentum and temperature equations

$\frac{\partial \tilde{\mathbf{u}}}{\partial t}+(\tilde{\mathbf{u}} \cdot \nabla) \tilde{\mathbf{u}}=-\frac{\nabla\left(\tilde{p}+p_{\infty}\right)}{\rho}-\nabla \cdot \boldsymbol{\tau}_{\boldsymbol{s}}+2 \boldsymbol{\omega} \times \tilde{\mathbf{u}}+g \frac{\left(\tilde{\theta}-\theta_{0}\right)}{\theta_{0}}+\overline{\mathbf{F}}$

$\frac{\partial \tilde{\theta}}{\partial t}+(\tilde{\mathbf{u}} \cdot \nabla) \tilde{\theta}=-\nabla \cdot \boldsymbol{q}_{s}$

are solved. In these equations, $\tilde{\mathbf{u}}=\left[\tilde{u_{1}}, \tilde{u_{2}}, \tilde{u_{3}}\right]$ is the filtered velocity field. Further, $\tilde{\theta}$ is the filtered potential temperature field, and $\theta_{0}$ is the background adiabatic base state. The pressure gradient is split into a mean background pressure gradient $\nabla p_{\infty}$ driving the mean flow, and a fluctuating component $\nabla \tilde{p}$. The very high Reynolds numbers in the atmospheric boundary-layer flow combined with typical spatial resolutions in LES justify the omission of resolved effects of viscous momentum transfer and diffusive heat transfer. Instead, these are represented by modeling the subgrid-scale stress tensor $\tau_{s}$ and the subgrid-scale heat flux $q_{s}$ originating from spatially filtering the original governing equations (Allaerts and Meyers, 2015). Coriolis effects are included through the angular velocity vector $\boldsymbol{\omega}=\Omega \sin \phi$, where $\Omega$ is the earth's rotation and $\phi$ is the latitude of the wind farm. Thermal buoyancy is represented by $g\left(\tilde{\theta}-\theta_{0}\right) / \theta_{0}$, with $g$ the gravitational acceleration, $\tilde{\theta}$ the filtered potential temperature and $\theta_{0}$ a reference temperature. The effect of the sea surface is included using a wall-stress model, corresponding to a logarithmic velocity profile with a roughness length $z_{0}$ (Bou-Zeid et al., 2005). Finally, $\overline{\mathbf{F}}$ represents any remaining body forces (e.g. by wind turbines) on the flow.

Spatial discretization is performed in the horizontal and span-wise directions by using pseudo-spectral schemes, while vertical fourth-order energy-conservative finite differences are used in the vertical direction. The equations are marched in time using an 
explicit fourth-order Runge-Kutta scheme, and grid partitioning is achieved through a scalable pencil decomposition approach. Subgrid-scale stresses are modeled with a standard Smagorinsky model with Mason and Thomson wall damping (Allaerts and Meyers, 2015).

\subsection{Structural solver}

Deformation of the turbine blade and tower is employed by a finite-element floating frame of reference formulation (Shabana, 2013). Each element is described by reference coordinates which specify its position and orientation, and elastic coordinates that define its deformation with respect to the body coordinate system. Bryant angles are used to describe the orientation of the rotor's body reference frame, however only the rotation of the turbine rotor is assumed to contribute to its dynamic behaviour. Deformations along the tilting, yawing and pre-coned axis are taken into account quasi-statically. The governing equation for the system can be written as (Shabana, 2013)

$\mathbf{M}(\mathbf{q}) \ddot{\mathbf{q}}+\mathbf{C} \dot{\mathbf{q}}+\mathbf{K}(\mathbf{q}) \mathbf{q}+\phi_{q}^{T} \lambda=Q_{a}+Q_{g}+Q_{v}$

$\phi(\mathbf{q})=0$

where, M, C, K, are the mass, damping and stiffness matrices respectively, computed using the structural specifications of the Siemens SWT-2.3-93 turbines. The vector q represents the generalized coordinates, while $\dot{\mathbf{q}}$ and $\ddot{\mathbf{q}}$ represent their first and second time derivatives. $\phi_{q}$ and $\boldsymbol{\lambda}$ are the constrain Jacobian matrix and Lagrange multipliers, respectively, and $\boldsymbol{Q}_{\boldsymbol{g}}$ represents the gravitational loads acting on the rotor and tower elements. The vector $\boldsymbol{Q}_{a}=\left[\mathbf{F}_{\mathbf{A}}^{\mathrm{rtr}} \mathbf{F}_{\mathrm{A}}^{\mathrm{twr}}\right]$ contains the aerodynamic loads evaluated at the rotor and tower nodes, as described in section 3.3. Finally, $\boldsymbol{Q}_{\boldsymbol{v}}$ is composed of the Coriolis and gyroscopic loads (Shabana, 2013). Further details regarding the coordinate system used and the derivation of the equations of motion is given in Appendix A.

To solve the equations of motion, first an eigenvalue problem is solved without damping and external loading to extract the mode shapes and natural frequencies of the structure. Then, the order of the system is reduced by a common modal transformation technique. Hence, the rotor blades are represented by 6 modes (two flap wise, two edgewise, one torsional and one axial) and the tower is represented by 4 modes( two side-to-side and two fore-aft). Finally, the reduced order system is integrated in time by using the generalized- $\alpha$ method, with a time-step of $0.01 \mathrm{~s}$ and spectral radius of 0.9 for low numerical damping (Arnold et al., 2007).

\subsection{Turbine Model}

As it's computationally prohibitive to fully resolve a wind turbine structure and its forces, actuator methods have been extensively used in research to parameterize wind turbine forces onto the flow grid. The most widely used amongst these models is the Actuator Disc Model (ADM) and the Actuator Line Model (ALM) (Churchfield et al., 2017). While the ALM provides the most accurate parametrization, it suffers from a limitation that the movement of the actuator line tip over a time step is limited 
to the size of a cell. To overcome this limitation, an Actuator Sector Model (ASM) was developed, which swept the rotor forces across a sector area and hence allowed for coarser time steps (Storey et al., 2015). The ASM was later extended to an Aeroelastic Actuator Sector Model (AASM) by incorporating two-way Fluid Structure Interaction (FSI) coupling, to account for structural deformations (Vitsas and Meyers, 2016).

In this work, the Siemens 2.3 MW turbines are modeled by using the AASM, coupled with the nonlinear flexible multibody dynamics model described in the previous section. Since the LES computations are more intensive than integrating the structural equations, a sub-cycling process is employed, for which the aeroelastic coupling scheme is shown in Figure 4. The relative velocity $V_{r e l}$ is evaluated at each airfoil element along the blade based on the induced velocity field $\hat{u}_{x}$ at the airfoil's deflected position, and on the blade's out-of-plane and in-plane motion represented by $q_{O_{O P}}$ and $q_{i P}$ respectively. The relative velocity $V_{r e l}$ also includes the effect of rotor angles (yaw, tilt and precone) by using rotation matrices to transform the incoming flow field. The flow angle $\phi$ is then determined from the involved velocity triangle, which comprises of the the pitch angle $\beta$, the torsional deflection $\tau$ and the angle of attack $\alpha$. The lift $L, \operatorname{drag} D$ and pitching moment $M$ at each airfoil section is then determined using 2D airfoil look-up tables, and used to evaluate the aerodynamic forces $\mathbf{F}_{\mathbf{A}}^{\mathbf{m}}$ comprising of the normal, tangential and span-wise forces, $F_{N}^{m}, F_{T}^{m}$ and $F_{S}^{m}$. This is done at every sub-cycle $m$, and serves as an input to the equations of motion given by equation 3, which are subsequently solved at each sub-step. The forces are then spatially and temporally filtered to obtain the body forces $\mathbf{F}$, which serve as an input to the flow solver in equations 1 . Further details of the coupling of the turbine model, flow solver and filtering are given in Appendix B.

The pitch and rotational speed of all the turbines are controlled using an implementation of the DTU wind energy controller (Hansen and Henriksen, 2013). A comparison of the simulated power output and thrust in SP-Wind using AASM under the influence of a range of uniform velocities against reference data for the Siemens 2.3 MW turbines is shown in Figure 5. Slight differences can be seen in both the simulated power and thrust, which can be attributed to the coarse grid resolution across the turbine blades in SP-Wind, which was chosen based on previous wind farm simulations for computational efficiency (Vitsas and Meyers, 2016; Munters and Meyers, 2018).

\section{Recreating the inflow conditions at Lillgrund}

\subsection{Precursor database}

The turbulent inflow conditions for wind-farm inflow are obtained from the publicly available precursor data from the TotalControl Flow database (Munters et al., 2019a, b, c, d). The precursor data contains unsteady three-dimensional flow data of an unperturbed atmospheric boundary layer (i.e. without the influence of turbines). The database comprises of two Pressure Driven Boundary Layers (PDBL) and three Conventionally Neutral Boundary Layers (CNBL), spanning different surface roughness lengths and boundary layer heights. Specifications of the five different boundary layers are given in Table 1, while their flow profiles are shown in Figures 6 and 7. The database has previously been used in a study to determine the effect of CNBL height on wind-farm performance (Sood et al., 2020). A stream-wise slab of the velocity and temperature field was stored to disk when running the precursor, and is later introduced in the wind-farm domain by means of body forces in a so-called fringe region 

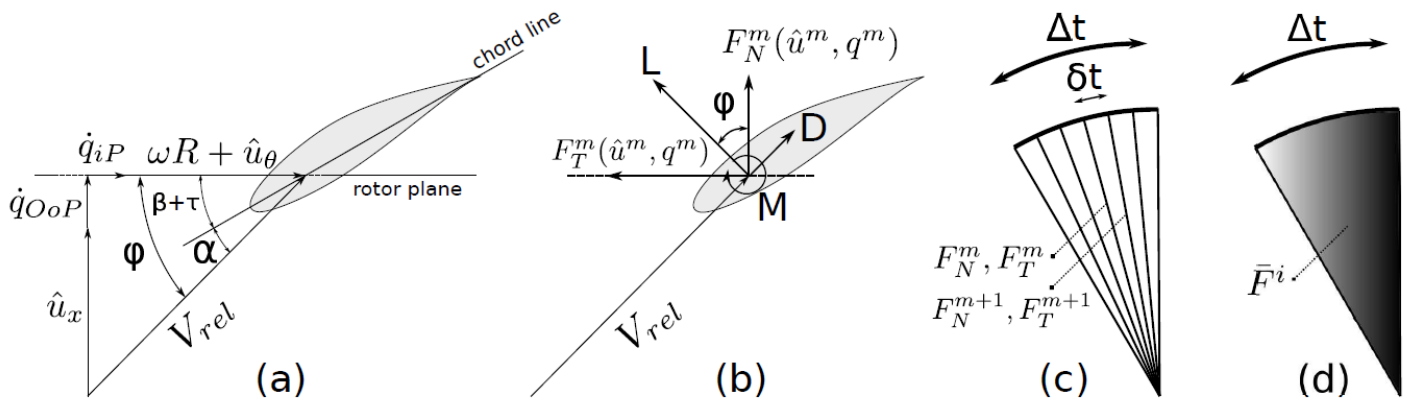

Figure 4. The AASM comprises of the steps: (a) evaluation of the angle of attack from the airfoil's cross-section velocity triangle and blade's motion, (b) the local cross-section forces are computed from 2D airfoil data, (c) the blades sweep a sector area using a sub-cycling scheme, and (d) the sector area forces are time-filtered. (d) denotes that more weight is given to the forces in the end position. Figure taken from Vitsas and Meyers (2016).

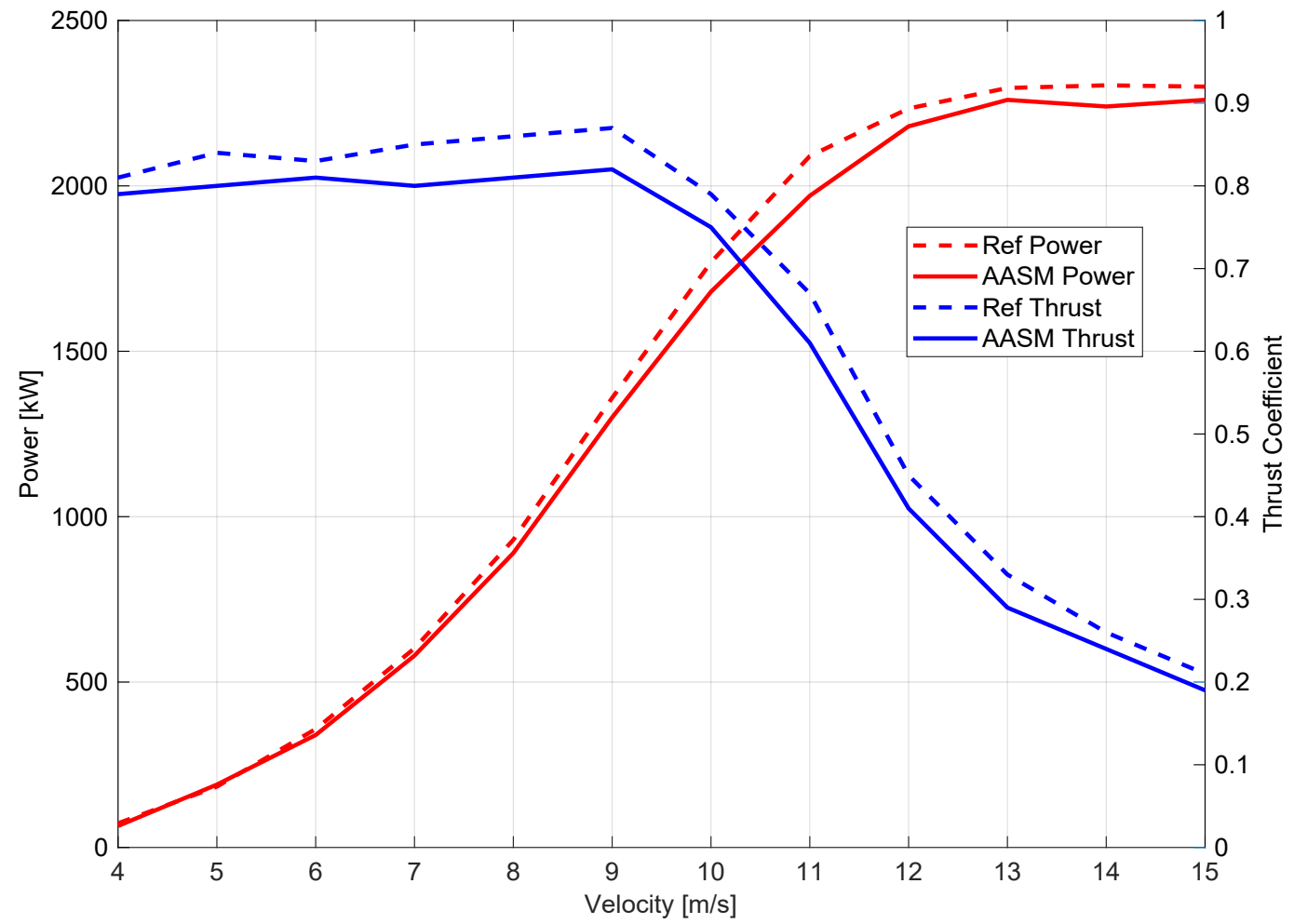

Figure 5. Comparison of simulated power and thrust coefficient in SP-Wind using the AASM against reference data (Göçmen and Giebel, 2016) 
https://doi.org/10.5194/wes-2021-153

Preprint. Discussion started: 20 January 2022

(c) Author(s) 2022. CC BY 4.0 License.
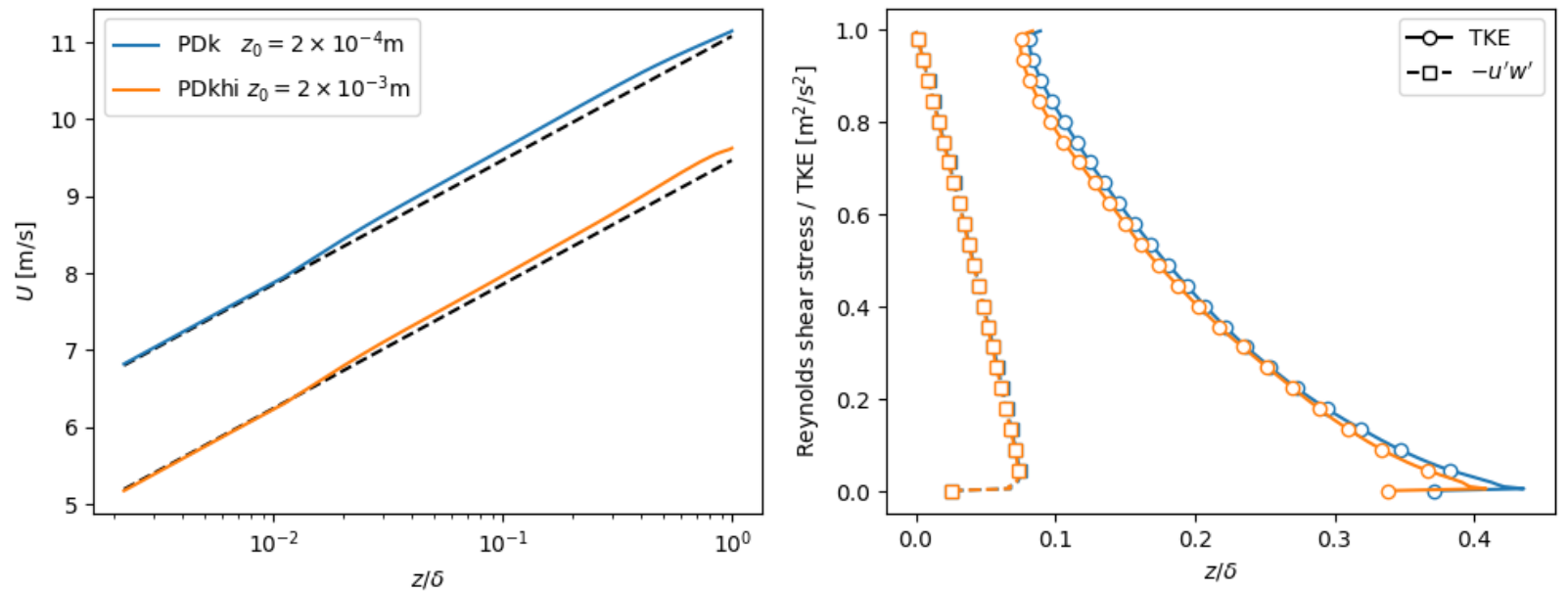

Figure 6. Flow profiles for PDBL cases. Left: Mean velocity. Dashed lines indicate log-law profiles. Right: resolved Reynolds shear stress and turbulent kinetic energy.
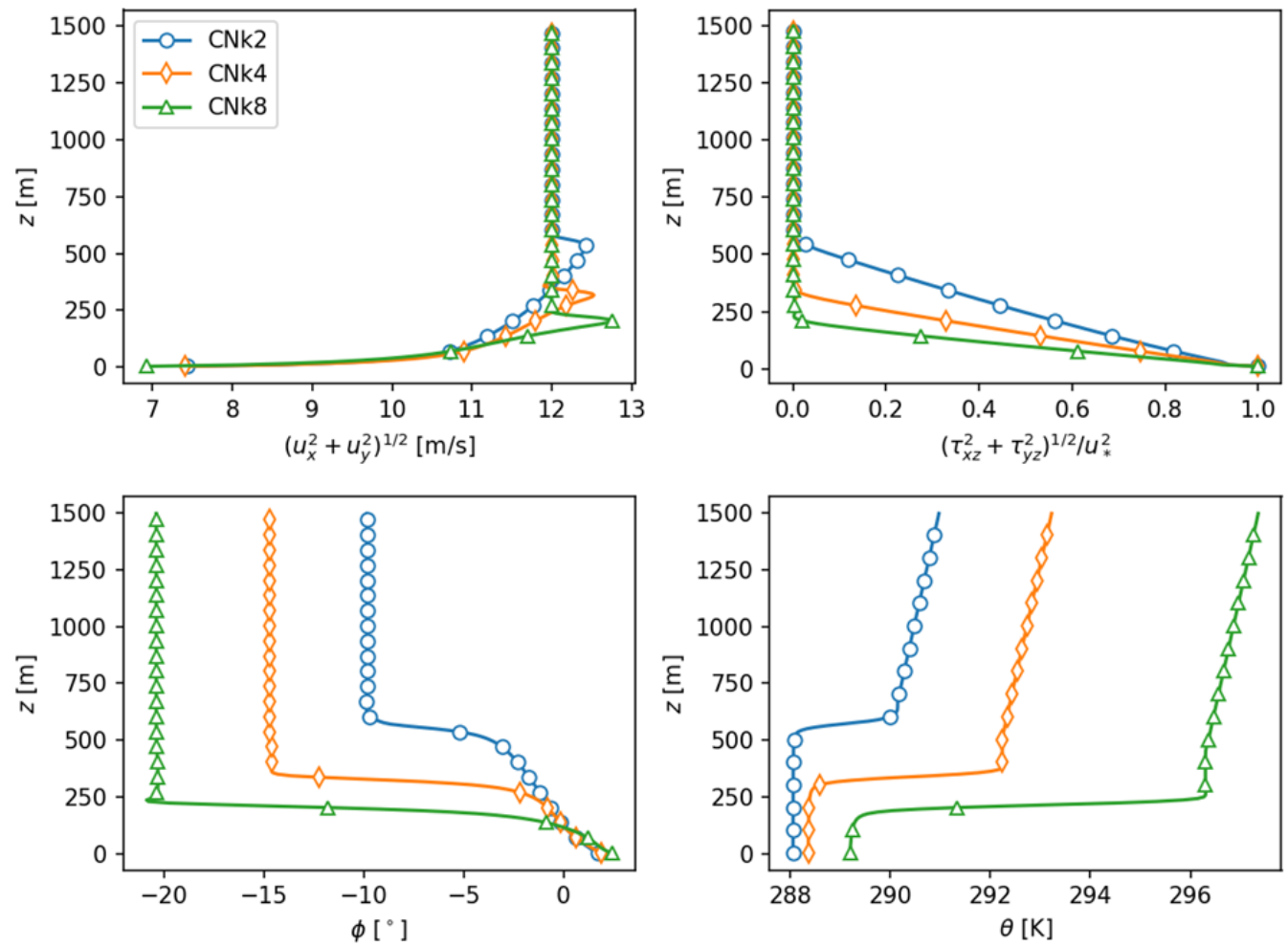

Figure 7. Flow profiles for CNBL cases. Top left: Horizontal Velocity. Top right: Total (Resolved + Subgrid) shear stress. Bottom left : Wind veer. Bottom right : Potential temperature. Figure taken from Sood et al. (2020). 
Table 1. Specifications of the TotalControl flow database (Munters et al., 2019a, b, c, d)

\begin{tabular}{lccc}
\hline Case & Boundary layer height & Surface Roughness & Capping Inversion strength \\
\hline$P D k$ & $1500 \mathrm{~m}$ & $2 \times 10^{-4} \mathrm{~m}$ & - \\
$P D k h i$ & $1500 \mathrm{~m}$ & $2 \times 10^{-5} \mathrm{~m}$ & - \\
$C N k 2$ & $500 \mathrm{~m}$ & $2 \times 10^{-4} \mathrm{~m}$ & $2 \mathrm{~K}$ \\
$C N k 4$ & $250 \mathrm{~m}$ & $2 \times 10^{-5} \mathrm{~m}$ & $4 \mathrm{~K}$ \\
CNk8 & $125 \mathrm{~m}$ & $2 \times 10^{-5} \mathrm{~m}$ & $8 \mathrm{~K}$ \\
\hline
\end{tabular}

(Stevens et al., 2014; Munters et al., 2016). To match the inflow conditions measured by the LiDAR measurement campaign, the data from the precursor data-set can be transformed to different flow conditions by re-scaling and shifting the flow variables. Using friction velocity $u_{*}$ for velocity scaling, different wind speeds can be attained by re-scaling the entire flow field by a different target friction velocity $u_{*}^{t}$. This is possible for offshore wind farms, as the solution is scale invariant at high Reynolds numbers. Additionally, in line with the classical outer layer similarity hypothesis (Townsend, 1976), for offshore atmospheric boundary layers at high Reynolds numbers the roughness elements are much smaller than the boundary-layer height, and hence the roughness acts merely to increase surface stress without any structural changes in the flow (Castro, 2007; Jiménez, 2004). The effect of a different target roughness lengths $z_{0}^{t}$ can thus be imposed by applying an offset on the mean flow in line with the difference in surface roughness. Hence, denoting the imposed reference friction velocity and roughness length in the current cases by $u_{*}^{r}$ and $z_{0}^{r}$ respectively, the flow can be re-scaled and shifted as

$205 u^{t}(x, t)=u_{*}^{t}\left[\frac{u^{r}(x, t)}{u_{*}^{r}}+\frac{1}{\kappa} \ln \frac{z_{0}^{r}}{z_{0}^{t}} \mathbf{e}_{\mathbf{1}}\right]$

Scaling the velocity also leads to scaling of the time scales, according to the following equation

$\Delta t^{t}=\frac{\Delta t}{u_{*}^{t}} u_{*}^{r}$

\subsection{Optimization framework}

210 While SP-Wind does support changing wind directions during a simulation run (Munters et al., 2016), each simulation is restricted to a single wind direction $\theta$ and a time frame of 75 minutes to limit computational costs. Thus, the available inflow data from the measurement campaign was divided into numerous 75 minutes overlapping time windows. Defining $X$ as the LES data-set index corresponding to the five available precursor cases listed in Table 1, and $Y$ as the index of a 75 minute time block extracted from the available LiDAR database, two distance metrics can be used to determine the differences between the 
LiDAR data-set and the available LES flow data-sets. These metrics are defined as,

$\boldsymbol{d}_{1}(\boldsymbol{X}, \boldsymbol{Y})=\left\|\overline{\boldsymbol{u}}_{X}-\overline{\boldsymbol{v}}_{Y}\right\|_{\boldsymbol{w}}^{2}$

$d_{2}(X, Y)=\left\|\operatorname{cov}\left(u_{X}\right)-\operatorname{cov}\left(v_{Y}\right)\right\|_{w}^{2}$.

In the distance metrics, $\boldsymbol{u}_{\boldsymbol{X}}$ represents a LES data-set $X$ from the TotalControl inflow database extracted at the range gate locations from a 3D velocity field, and $v_{Y}$ represents a LiDAR data-set $Y$, spanning a 75 minute time window from the measurement campaign. For both the LES and LiDAR data-sets, only the two horizontal velocity components are included in the distance metrics as vertical velocity measurements were not available in the LiDAR database. Rows in the two data-sets represent range gate locations from the measurement campaign, while columns represent time-series data. As per their definitions, $\boldsymbol{d}_{1}$ provides a measure of the difference between the time averaged profiles at range gate locations, while $\boldsymbol{d}_{2}$ is a difference between the co-variances of the two data-sets, accounting for spatial relationships. The metrics are also assigned weights according to a vector $\boldsymbol{w}$ as shown in Figure 8(c), giving highest preference to range gates spanning the hub height, followed by the remaining rotor area and finally the rest of the vertical domain. As discussed in Section 4.1, the available LES data-sets can be modified for each case $X$ by using the scaling and shifting parameters $u_{*}^{t} / u_{*}^{r}$ and $z_{0}^{t} / z_{0}{ }_{X}^{r}$, henceforth collectively referred to as the transformation vector $\boldsymbol{\zeta}=\left[u_{*}^{t}{ }_{X}, z_{0}^{t}\right]$. Therefore, the distance metrics $\boldsymbol{d}_{1}(X, Y, \boldsymbol{\zeta})$ and $\boldsymbol{d}_{2}(X, Y, \boldsymbol{\zeta})$ can be determined over the entire 6 month measurement campaign between each LES data-set $X$ and a LiDAR data-set $Y$, for different combinations of the transformation parameters. The sum of both the metrics can be used as a measure of similarity between the LES and LiDAR data, with lower values indicating greater similarity. Thus for each data-set $X$, a minimization problem can be defined to determine the transformation vector $\zeta$ and the time window $Y$ from the measurement campaign which returns the least distance between the LES data and LiDAR measurements, indicating highest similarity. The cost function of the optimization problem can be defined as,

$\min _{\zeta, Y} \boldsymbol{d}_{\mathbf{1}}(X, Y, \boldsymbol{\zeta})+\boldsymbol{d}_{\mathbf{2}}(X, Y, \boldsymbol{\zeta})$

and is solved using the SLSQP solver from the SciPy Python package (Virtanen et al., 2020). After sweeping through the entire measurement campaign, five unique time windows of 75 minute length each, corresponding to five different LES flow realizations which best matched the LiDAR data are obtained. The first three matches are obtained by transforming the PDk TotalControl LES data-set, while the fourth and fifth matches are obtained from the CNk4 and CNk8 data-set respectively, without any transformations. While additional matches were identified, which also contained flow realizations obtained from the $P D k h i$ and $C N k 2$ LES data-sets, we restrict further analysis to the best five cases with highest similarity due to computational limitations. A comparison of the mean vertical profiles at range gate location for these cases are shown in Figure 8 and their specifications are outlined in Table 2 . A comparison of the average turbulence intensity across the range gates spanning the rotor area is also presented in Table 3. From Figure 8, it can be seen that all the selected LES cases match the LiDAR measurements very well across the rotor area. Larger deviations in total mean wind speed can be observed at heights above the rotor tip, which 
https://doi.org/10.5194/wes-2021-153

Preprint. Discussion started: 20 January 2022

(c) Author(s) 2022. CC BY 4.0 License.
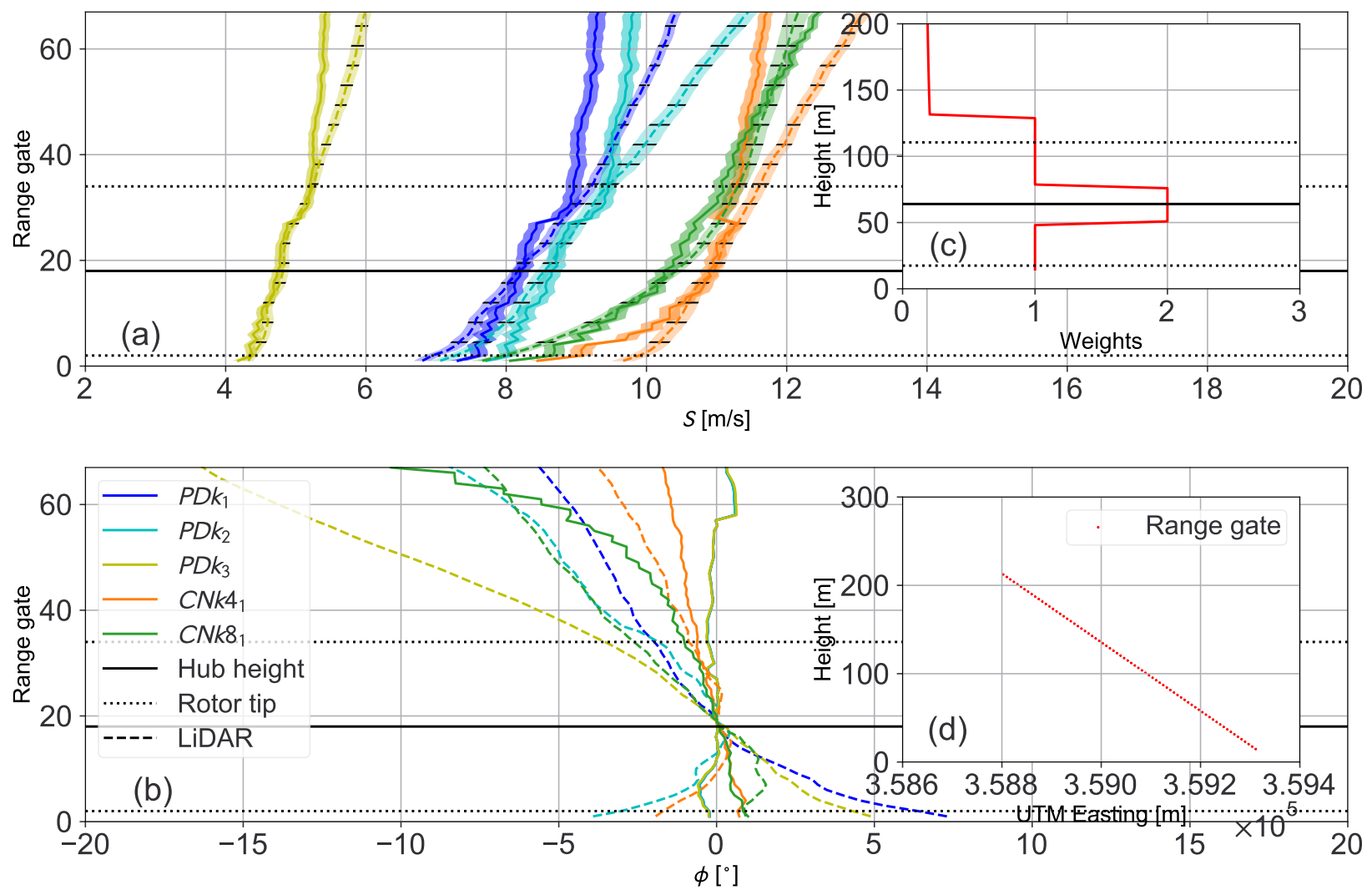

Figure 8. Comparison of vertical mean total velocity profile (a) and wind veer (b) between the selected LES and corresponding LiDAR data. (c) Height varying weights used for the distance metrics in the minimization problem. (d) Locations of 72 range gates, with range gate 1 being the lowest and closest to the turbine B08, and 72 being the highest and furthest away. Shaded area represents 95 percent confidence intervals on the mean.

Table 2. Specifications of selected validation cases

\begin{tabular}{lccccc}
\hline Case & Measurement campaign time & Friction velocity $\left(u_{*}\right)$ & Surface roughness $\left(z_{0}\right)$ & hub height wind direction $(\theta)$ & hub height velocity $(S)$ \\
\hline$P D k_{1}$ & $2019-12-23, \mathrm{~T} 01: 14: 44$ & $0.267 \mathrm{~ms}^{-1}$ & $2.17 \times 10^{-4}$ & $119^{\circ}$ & $8.2 \mathrm{~ms}^{-1}$ \\
$P D k_{2}$ & $2019-12-18, \mathrm{~T} 18: 48: 04$ & $0.280 \mathrm{~m} \mathrm{~s}^{-1}$ & $2.00 \times 10^{-4}$ & $243^{\circ}$ & $8.5 \mathrm{~ms}^{-1}$ \\
$P D k_{3}$ & $2019-09-24, \mathrm{~T} 18: 01: 46$ & $0.168 \mathrm{~m} \mathrm{~s}^{-1}$ & $5.60 \times 10^{-4}$ & $110^{\circ}$ & $4.8 \mathrm{~ms}^{-1}$ \\
$C N k 4_{1}$ & $2020-01-29, \mathrm{~T} 05: 40: 36$ & $0.280 \mathrm{~ms}^{-1}$ & $2.00 \times 10^{-4}$ & $251^{\circ}$ & $222^{\circ}$ \\
$C N k 8_{1}$ & $2020-01-07, \mathrm{~T} 17: 46: 26$ & $0.280 \mathrm{~ms}^{-1}$ & $2.00 \times 10^{-4}$ & $10.8 \mathrm{~ms}^{-1}$ \\
\hline
\end{tabular}


Table 3. Turbulence Intensity (TI) comparison of validation cases, averaged over the rotor area

\begin{tabular}{lcc}
\hline Case & LiDAR TI [\%] & LES TI [\%] \\
\hline$P D k_{1}$ & 5.52 & 6.31 \\
$P D k_{2}$ & 5.99 & 6.27 \\
$P D k_{3}$ & 6.64 & 6.83 \\
$C N k 4_{1}$ & 5.12 & 5.59 \\
$C N k 8_{1}$ & 5.86 & 5.73 \\
\hline
\end{tabular}

can be attributed to the preference given to the hub height and rotor disc area through weights in the optimization problem. The PDBL simulations have the largest error when comparing the mean veer between the LES and LiDAR measurements, as by definition, the PDBL simulations have zero veer and are hence incapable of representing a veered flow. While the CNBL simulations do include veer, the TotalControl precursor database was not designed to cover large veer conditions, thus still leading to errors when compared to the veer in measurement data. Nevertheless, the absolute error never exceeds more than 7 degrees over the rotor area for any of the cases at a given range gate. Good comparison is also seen between the measured and simulated turbulence intensity, with the maximum error never exceeding $1 \%$.

\subsection{Numerical setup}

The simulation domain in SP-Wind has a size of $16 \times 16 \times 1.5 \mathrm{~km}^{3}$ in the stream-wise, span-wise, and vertical directions respectively. The grid resolution is $13.33 \times 13.33 \times 6.66 \mathrm{~m}^{3}$, resulting in a computational grid of $1200 \times 1200 \times 225=324 \times 10^{6}$ grid-points. Wind-farm simulations in SP-Wind are performed in a sequence of steps. First, the inflows from the previously generated TotalControl precursor database are made to advance in time in a domain without wind turbines, called the precursor domain. Concurrently, the flow is transformed to obtain the five cases identified in Table 2 and fed into a second domain, called the main domain, which contains wind turbines represented by the AASM. For each of the five cases, the Lillgrund wind-farm is rotated to simulate different wind directions. The flow is allowed to pass through the wind farm for 1800 time steps to account for start-up transients, after which data is collected for evaluating the performance of the farm for 9000 time steps. While the original precursor inflow database have a LES time step $\Delta t_{L E S}=0.5 \mathrm{~s}$, the time-step of the wind-farm simulations is altered due to the scaling of the velocity field, as per equation 6 . The multibody aeroelastic computations are performed with a smaller time step of $\Delta t_{M B}$, for a finer resolution of the structural loading. The general domain and time parameters of the simulations are summarized in Table 4. 
Table 4. Summary of the general domain parameters

\begin{tabular}{lcc}
\hline Domain size & $L_{x} \times L_{y} \times L_{z}$ & $16 \times 16 \times 1.5 \mathrm{~km}^{3}$ \\
Grid & $N_{x} \times N_{y} \times N_{z}$ & $1200 \times 1200 \times 225$ \\
Resolution & $\Delta_{x} \times \Delta_{y} \times \Delta_{z}$ & $13.33 \times 13.33 \times 6.66 \mathrm{~m}^{3}$ \\
Wind-farm spin-up time & $T_{\text {spin }}$ & $1800 \times \Delta t_{L E S}$ \\
Simulation time & $T$ & $9000 \times \Delta t_{L E S}$ \\
Structural time step & $\Delta t_{M B}$ & $0.02 \times \Delta t_{L E S}$ \\
\hline
\end{tabular}

\section{Results}

\subsection{Time averaged flow fields}

Time-averaged hub height flow fields for all the selected validation cases are shown in Figure 9. It can be seen that out of all the cases, $C N k 4_{1}$ has the highest inflow velocity and $P D k_{3}$ has the lowest, in accordance with results shown in Figure 8 . The different wind directions spanning the five cases lead to different operation states of the same turbines within the Lillgrund wind farm, due to changes in available hub height wind speed, but also due to individual turbines operating in a waked or un-waked condition as per the orientation of the upstream turbines. For instance, the cases $P D k_{1}$ and $C N k 8_{1}$ with wind directions $119^{\circ}$ and $222^{\circ}$ respectively, have a larger number of turbines operating under fully waked condition compared to the other three cases. This leads to a data-set which allows us to evaluate the performance of Lillgrund turbines when subjected to varying operating conditions.

\subsection{Performance comparison}

Comparison of the mean wind farm power output obtained from SP-Wind against the field measurements from Lillgrund is presented in Figure 10. As the sample size of comparison is limited, bootstrapping is used to determine the measure of accuracy of the computed means. Since a traditional bootstrap approach of randomly re-sampling the original time-series data is inappropriate for time seires with intrinsic correlation, the moving block bootstrap method is utilized (Kunsch, 1989). The length of individual blocks in the moving bootstrap method was set to 10 minutes, as a compromise between having enough bootstrap blocks from the measured time-series data, and keeping the block lengths large enough to ensure that individual blocks can be assumed to be independent from each other. Through a sensitivity study, it was determined that 1000 bootstrap iterations were sufficient to obtain converged uncertainty estimates for the mean. From Figure 10, it can be seen that the total farm power production in LES for the cases $P D k_{1}$ and $P D k_{2}$ are in good agreement with the measurement data, while larger errors can be seen for the other three cases. This is in accordance with the errors in the inflow profiles observed in Figure 8, where larger errors in mean inflow across the rotor area exist for the CNBL cases, and the $P D k_{3}$ case suffers from errors 

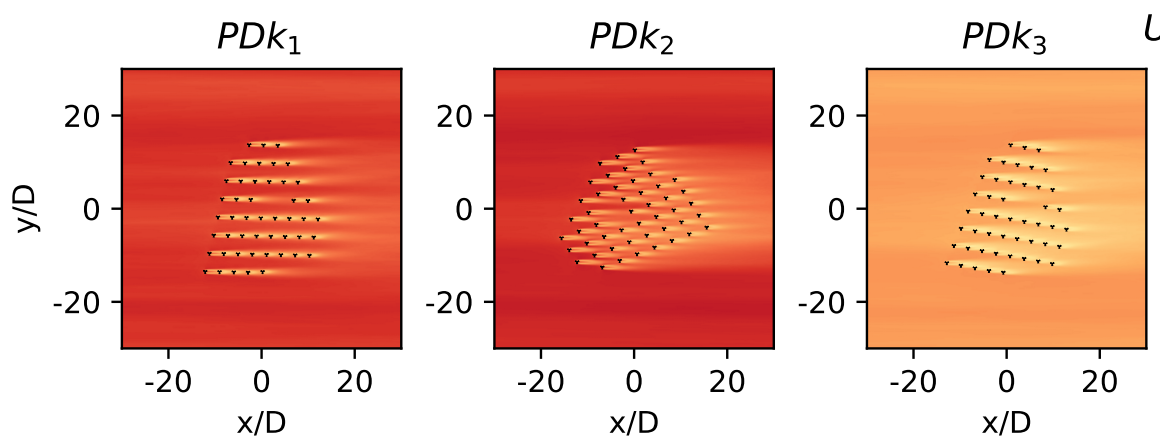

$U\left[m s^{-1}\right]$
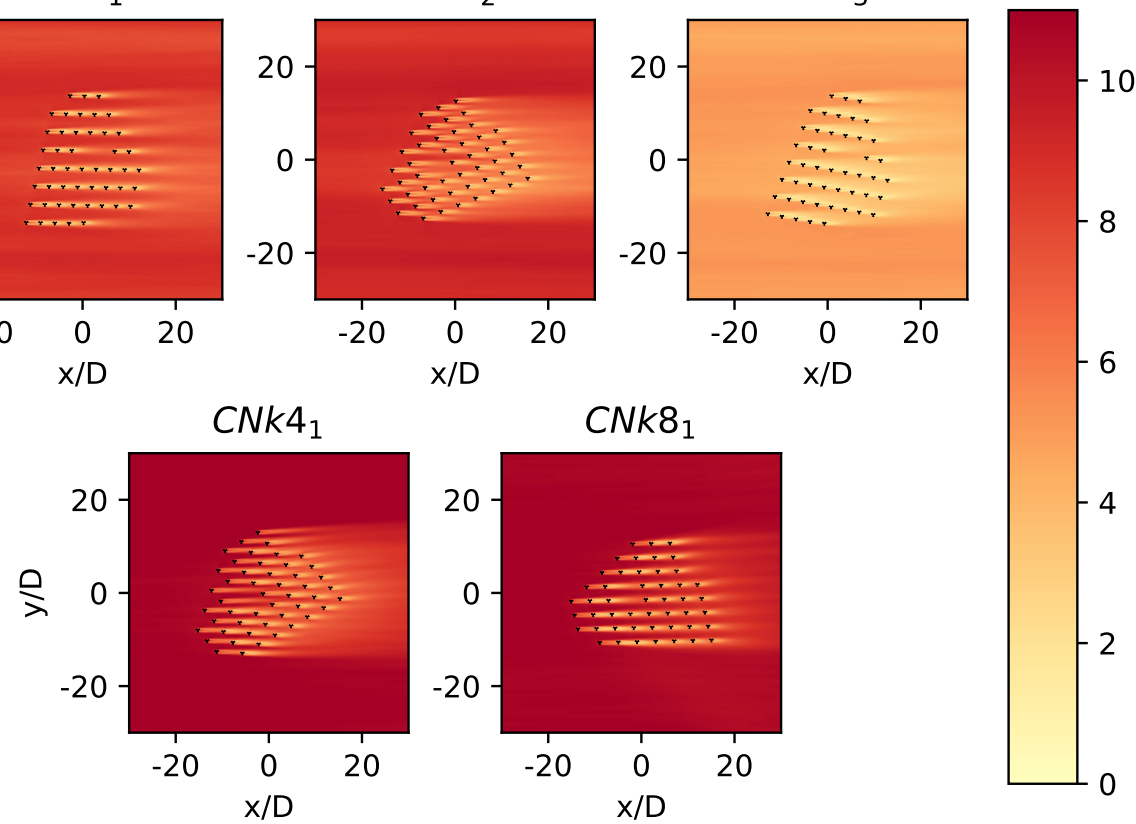

Figure 9. Time averaged stream-wise hub height velocity for all selected cases. Different wind directions are realized by rotating the entire wind-farm in the simulation domain and feeding the inflow velocity in the horizontal-x direction.

in wind veer. The distribution of individual turbine power production for the case $P D k_{1}$ is shown in Figure 11, including uncertainty estimates again determined by the moving bootstrap method. Individual turbine power trends across the farm show good agreement with field data, where similar trends are observed for power peaks and valleys for un-waked and waked turbines, indicating that on average the wind direction in the LES cases captures the real world field conditions during the time windows.

To further investigate the discrepancies in individual turbine power output across the five cases, we investigate the performance of the implemented controller in the wind farm. Figure 12 shows a comparison between the rotor speed and pitch actuation in SP-Wind against field measurements for all the turbines. It can be seen that while the performance of the pitch actuation and rotor speed controller show a good comparison in general across the five cases, larger errors can be seen for a few turbines in terms of both pitch and rotational speed. In particular, pitch measurements from the wind farm exhibit non-zero pitch angles for the majority of the turbines, even though they are operating in below rated conditions and should traditionally have zero pitch, as is the case for the turbines operating in the LES simulations. This demonstrates the differences between the actual field turbine controller and the one implemented in SP-Wind, as detailed information about the controller and all of 

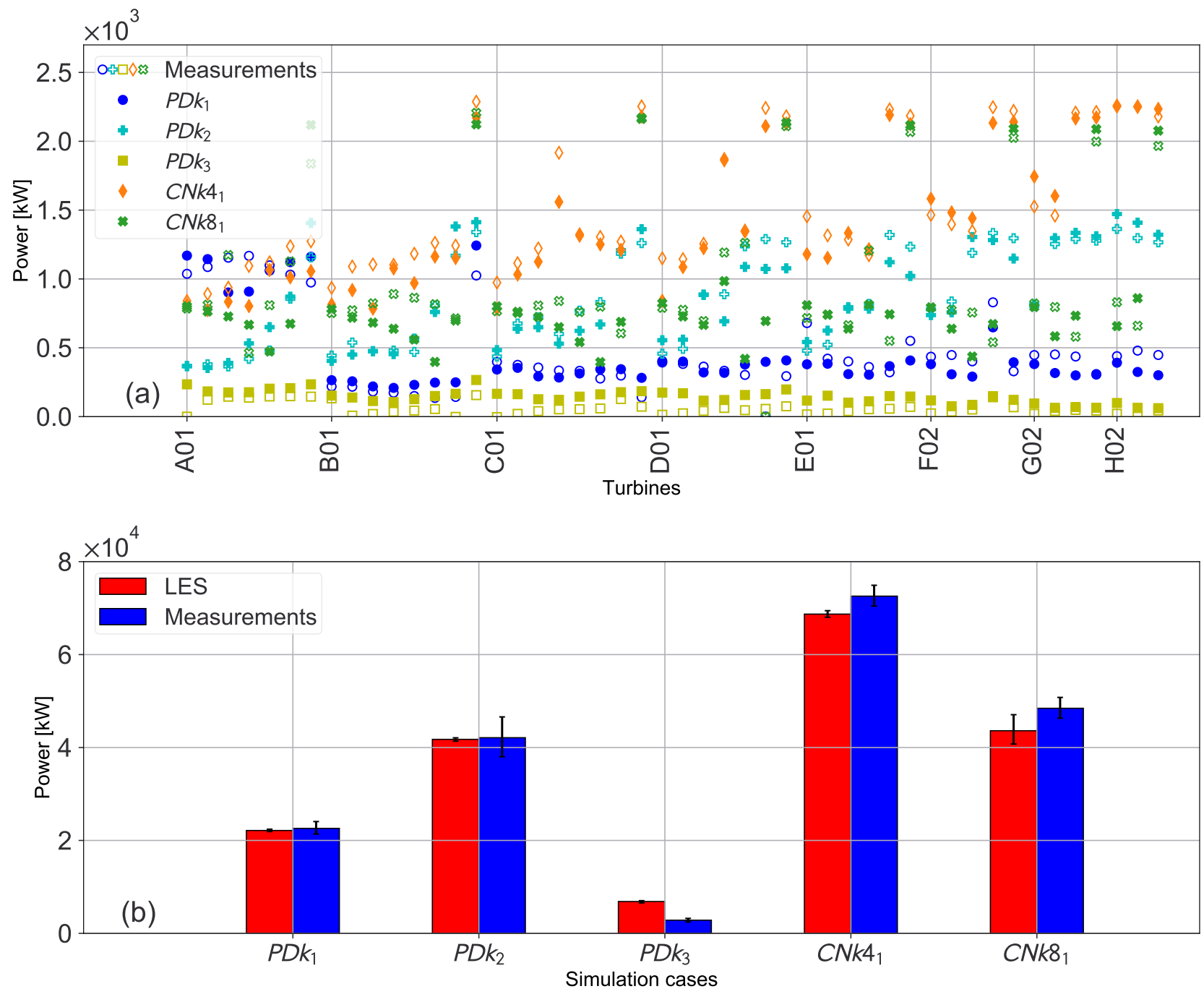

Figure 10. Comparison of LES time averaged power output of individual turbines (a) and total wind farm power output (b) against field SCADA measurements. Error bars represent 95 percent confidence intervals on the mean and are computed using the block-bootstrap method and time windows of $600 \mathrm{~s}$ and 1000 bootstrap samples. 


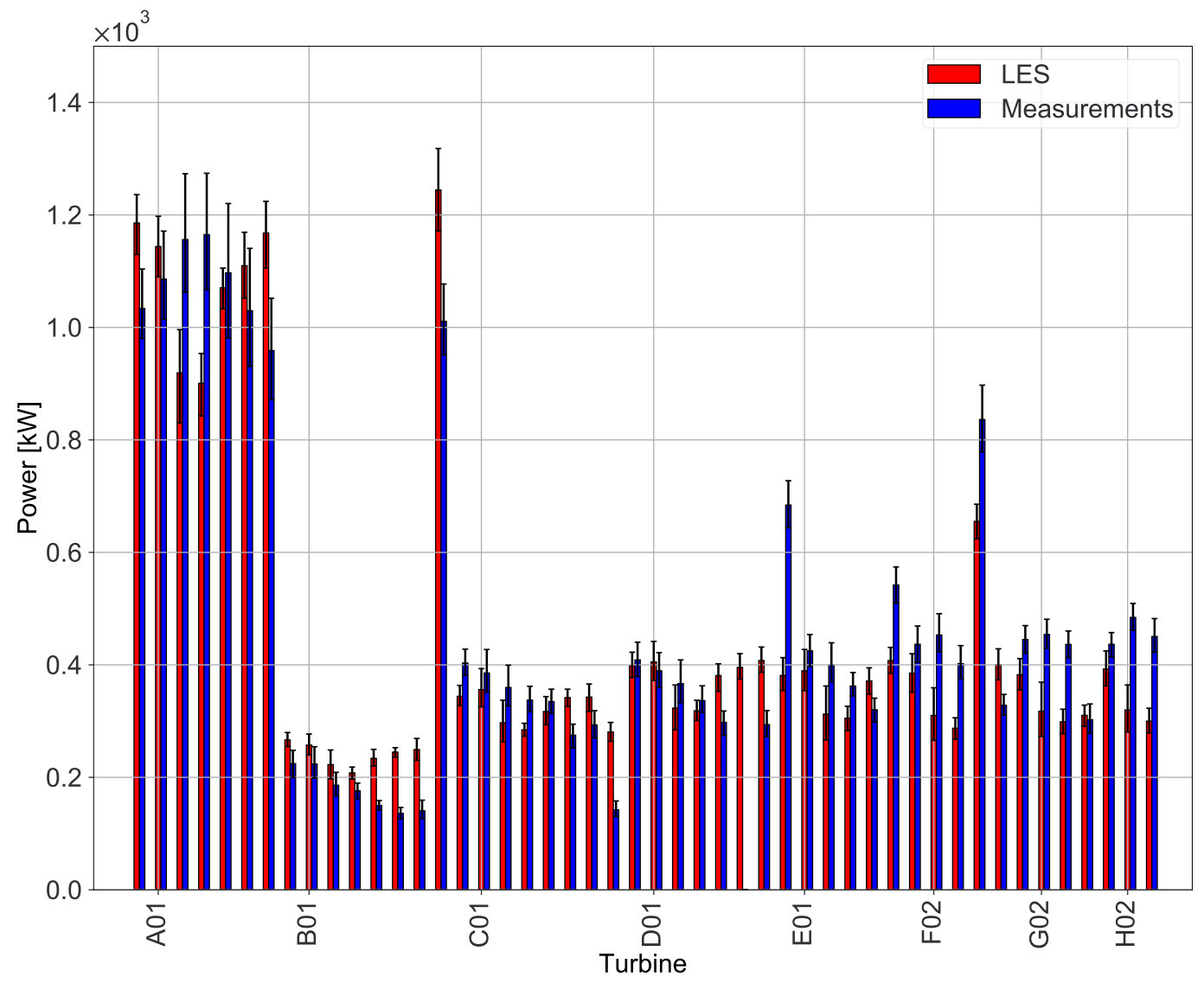

Figure 11. Bar plot of turbine power production for the case $P D k_{1}$. Error bars represent 95 percent confidence intervals on the mean and are computed using the block-bootstrap method and time windows of $600 \mathrm{~s}$ and 1000 bootstrap samples.

its modes of operation was not available. Larger pitch angles would lead to reduced power production for the turbines, which can be observed for the $P D k_{3}$ case with the maximum amount of turbines with non-zero pitch, leading to the larger errors in power production as observed in Figure 10. Another source of error worth investigating is the yaw misalignment between the simulated cases and the field measurements, which is presented in Figure 13. While all the turbines in the simulation domain of SP-Wind are statically aligned with the mean wind direction as identified for each time window and listed in Table 2 , it can be observed that this was not the case for the field turbines, which dynamically change their orientation in accordance with local wind direction changes within the farm, resulting in errors of turbine orientation. However, in majority of the cases the yaw misalignment error remains within \pm 5 degrees, causing minimal influence on the power production. Even for higher errors as observed for the $P D k_{1}$ case with a maximum error of -15 degrees for the B01 turbine, minimal influence on the power can 

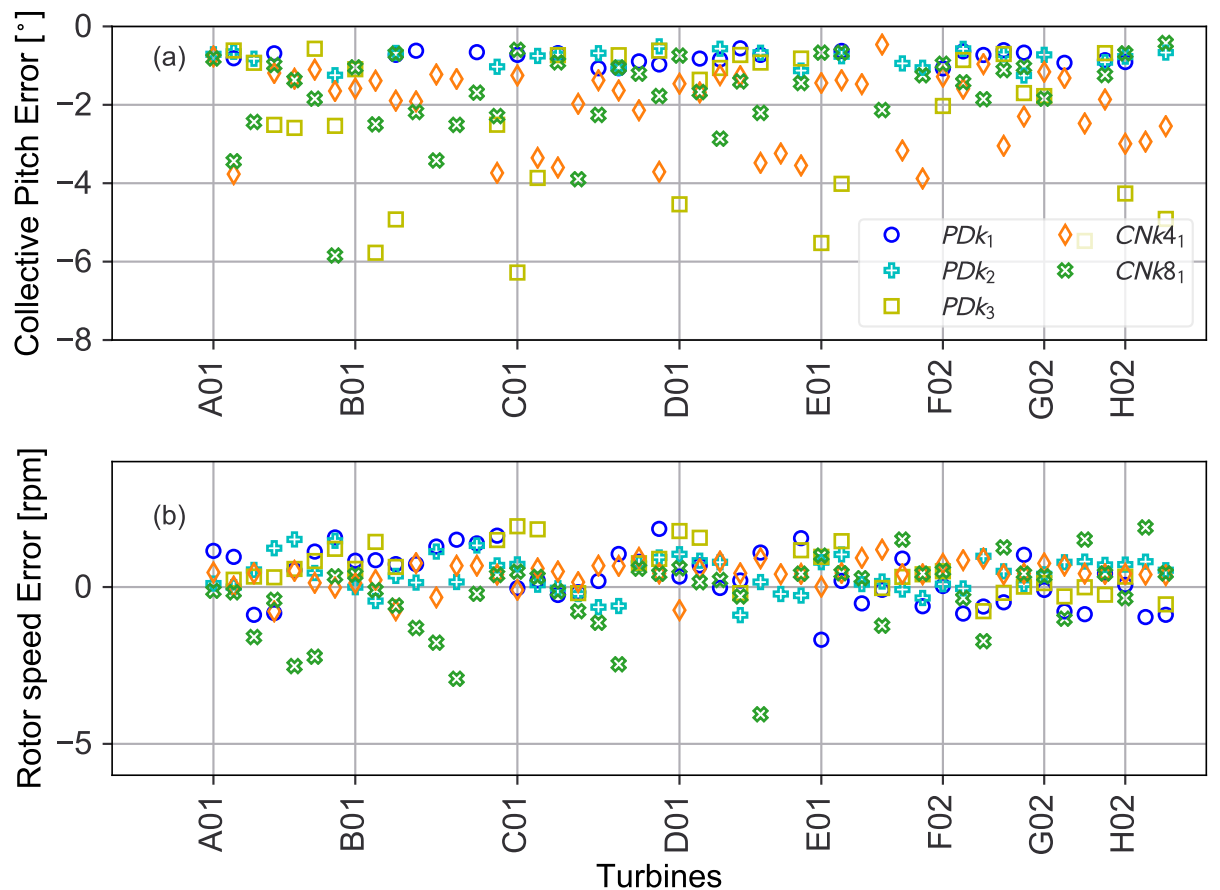

Figure 12. Errors between LES time averaged collective pitch (a) and rotational speed (b) of individual turbines and field SCADA measurements. Missing data points correspond to missing data.

be observed in Figure 10. This indicates that the errors in controller operation can be attributed to be the major contributor to the differences in power production.

Comparison of the mean flap-wise blade root bending moments for five of the 48 turbines from the Lillgrund wind-farm for which loading data was available is shown in Figures 14 and 15 for the PDBL and CNBL simulations, respectively. To determine the effect of fatigue, we use the damage equivalent loads (DELs) to compare the load histories of the same turbines across the LES and field measurement data. DEL is computed using the Palmgren-Miner rule and the Wöhler equation to account for accumulating fatigue damage caused to the wind turbine components by the fluctuating structural loads (Sutherland, 1999). The loads time series are counted and binned into individual cycles using the rainflow-counting algorithm (Socie and Downing, 1982), and for the wind turbine blades the components follow the Wöhler's curve with a slope coefficient equal to 10 (Freebury and Musial, 2000). The moving block bootstrap methodology is again utilized for evaluating the mean flap-wise moment and the corresponding DEL, with block lengths of 10 minutes and 1000 bootstrap iterations. Fatigue analysis was not conducted for the CNBL simulations, as the data was not logged consistently for the turbines in the time periods of these simulations, making the data unfit for rainflow analysis. Both the average blade root flap-wise moments and the corresponding DELs exhibit a good comparison in the trends of the PDBL simulations in Figure 14. For the $P D k_{2}$ case, while the loads of the un-waked upstream turbines B08, C08 and D08 show lower errors, significant errors are observed for the turbines B06 and B07, 


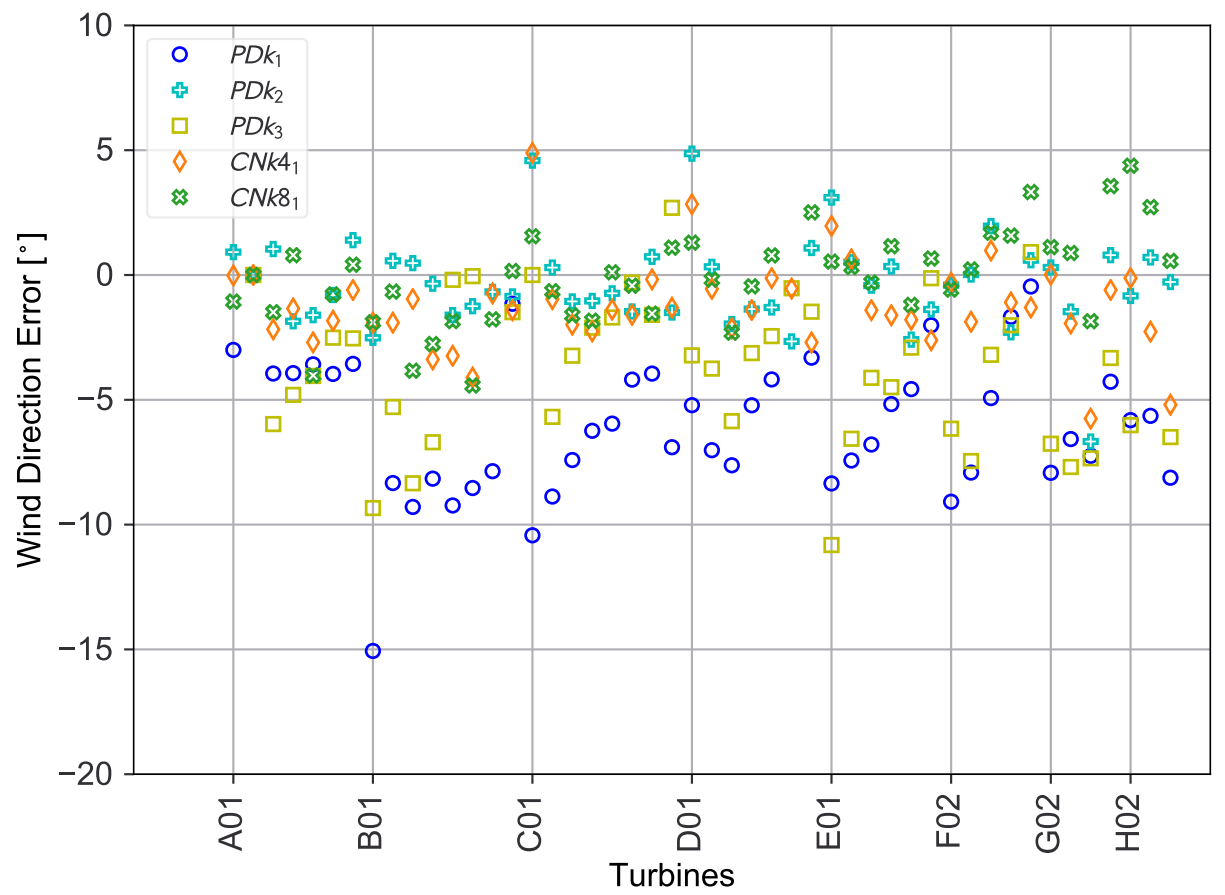

Figure 13. Errors between LES time averaged wind direction at individual turbines and field SCADA measurements. All turbines in the LES domain face the wind and have zero yaw misalignment. Missing data points correspond to missing data.

which are operating in a waked state. A similar observation can be made for the CNBL simulations in Figure 15. A possible explanation for the larger errors observed in the loads of the waked turbines could be the relatively coarse grid resolution utilized in SP-Wind when looking at the number of cells across the rotor diameter, such that not all relevant turbulent structures that contribute to loads are captured. In the current work, use of a finer grid for the simulations was not feasible, as the grid resolution of the wind-farm simulations is already quite high.

\subsection{Wake analysis}

Due to equipment failure, data from the wake measuring LiDAR's was unfortunately available for only two cases, $P D k_{3}$ and $C N k 4_{1}$, from the five selected validation time periods from the measurement campaign. Comparing wake recovery for the B06 turbine in the $C N k 4_{1}$ case in Figure 16, we see good agreement in wake location and recovery downstream from the turbines. It can be observed that while SP-Wind provides a good representation of the near wake region behind turbine B06, the far wake region characterized by a downstream distance greater than 4 rotor diameters exhibits higher errors. This can further be observed from the stronger wakes in SP-Wind in the far wake region for turbine C06, leading to a lower inflow velocity at turbine B05 and hence the underestimation of power production for turbine B05 as observed in Figure 10. Observing the wakes from the B05 turbine from $P D k_{3}$ in Figure 17, the effect of incorrectly representing the turbine orientation can be seen. While 
https://doi.org/10.5194/wes-2021-153

Preprint. Discussion started: 20 January 2022

(c) Author(s) 2022. CC BY 4.0 License.
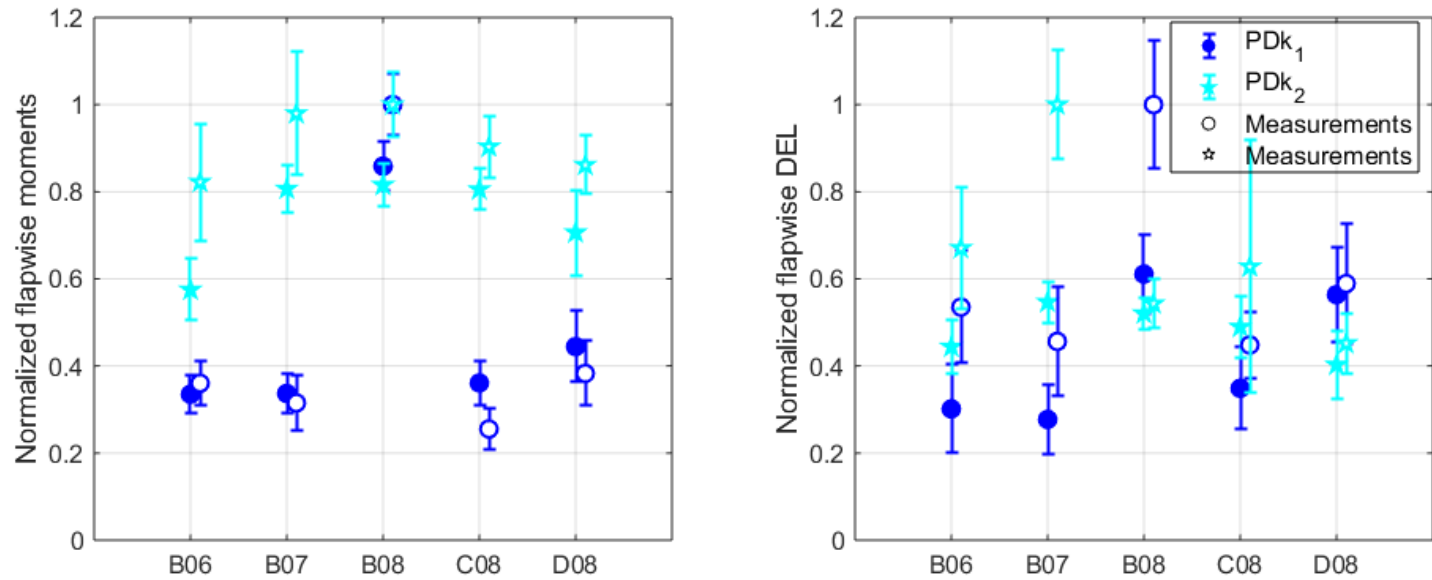

Figure 14. Comparison of LES time averaged blade root flap-wise moments (a) and DEL (b) against field measurements. Error bars represent 95\% confidence intervals on the mean. Results are normalized by maximum SCADA data for each case.
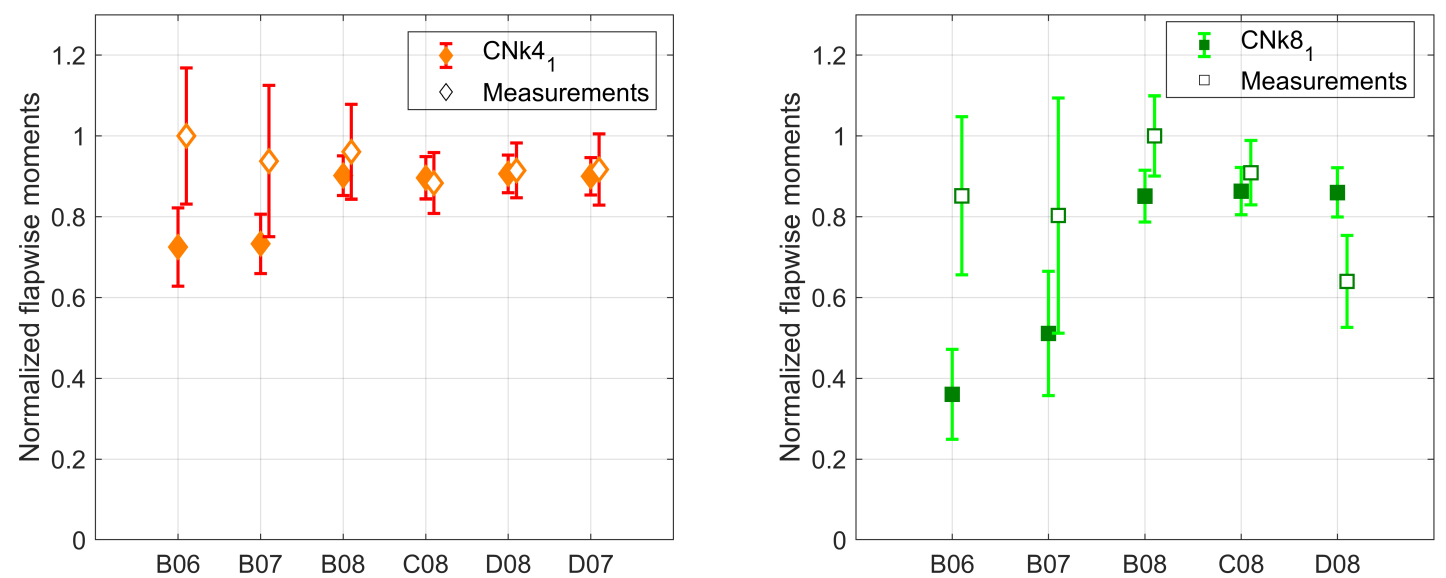

Figure 15. Comparison of LES time averaged blade root flap-wise moments for (a) $C N k 4_{1}$ (b) $C N k 8_{1}$ against field measurements. Error bars represent $95 \%$ confidence intervals on the mean. Results are normalized by maximum SCADA data for each case. 

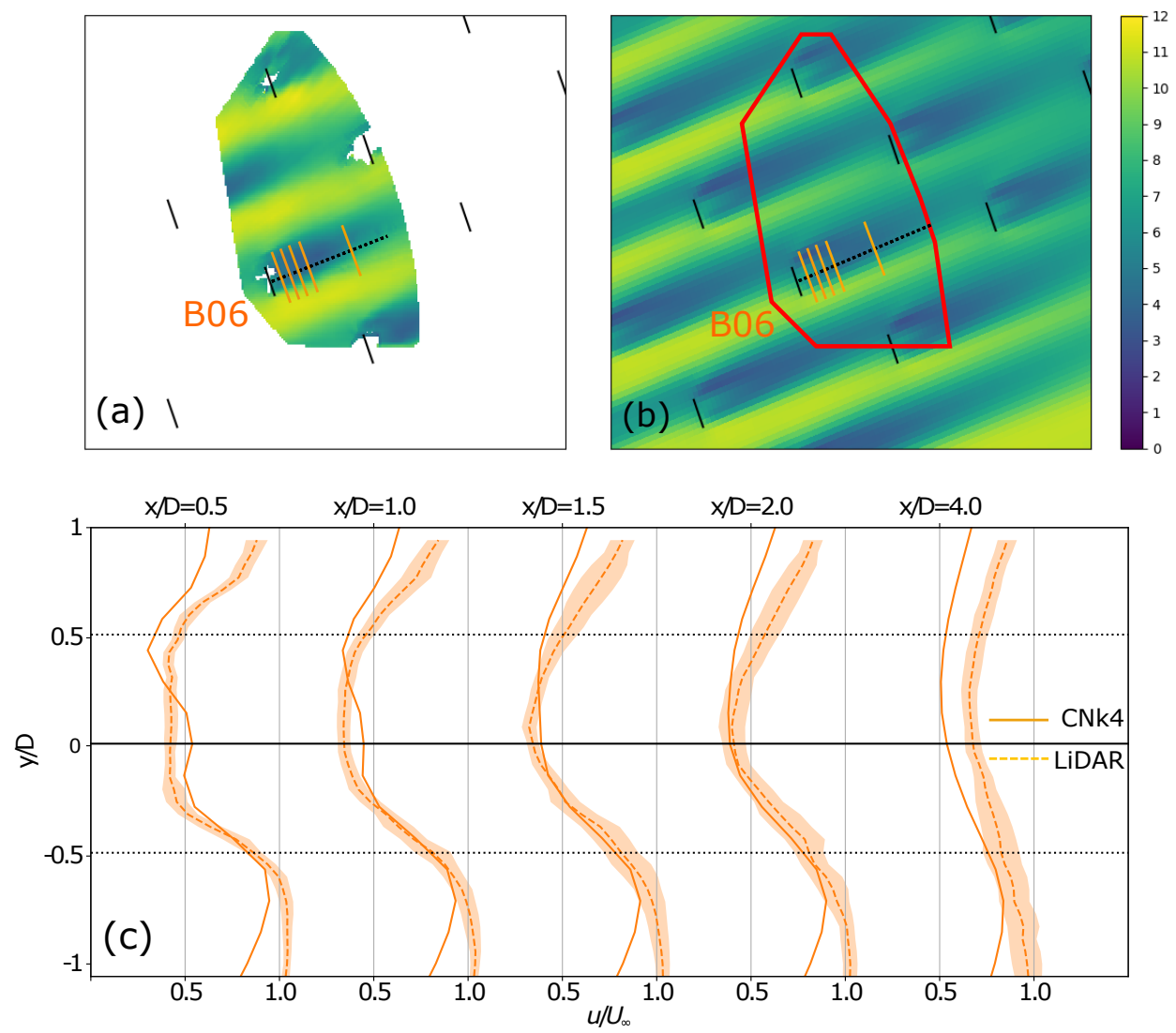

Figure 16. Comparison of LES time averaged velocity (b) against LiDAR wake measurements (a) for case $C N k 4_{1}$ and turbine B06.(c) Comparison of velocity wake deficit at downstream locations from turbine B06.

field measurements show a fully aligned configuration for turbines A05, B05 and C05, partial waked conditions are observed in SP-Wind, leading to a larger inflow velocity at turbine C05 and hence the greater reported power production.

\section{Discussion, conclusions and future work}

In this work, a validation study was conducted to compare SP-Wind, a high-fidelity Large Eddy Simulation solver, against field measurements obtained from the Lillgrund offshore wind-farm near the coast of Sweden. To recreate the atmospheric conditions at the Lillgrund site, a framework was developed to create the inflow conditions for the wind farm in the numerical domain by reusing a precursor database through scaling and shifting of the velocity. Thus, the cost intensive step of developing multiple precursor simulations for different atmospheric conditions spanning the duration of the measurement campaign was eliminated. Five time periods from the measurement campaign were selected for simulations in the LES environment. Upon comparison against field measurements, results from SP-Wind show good comparison in terms of the recreated inflow velocity 

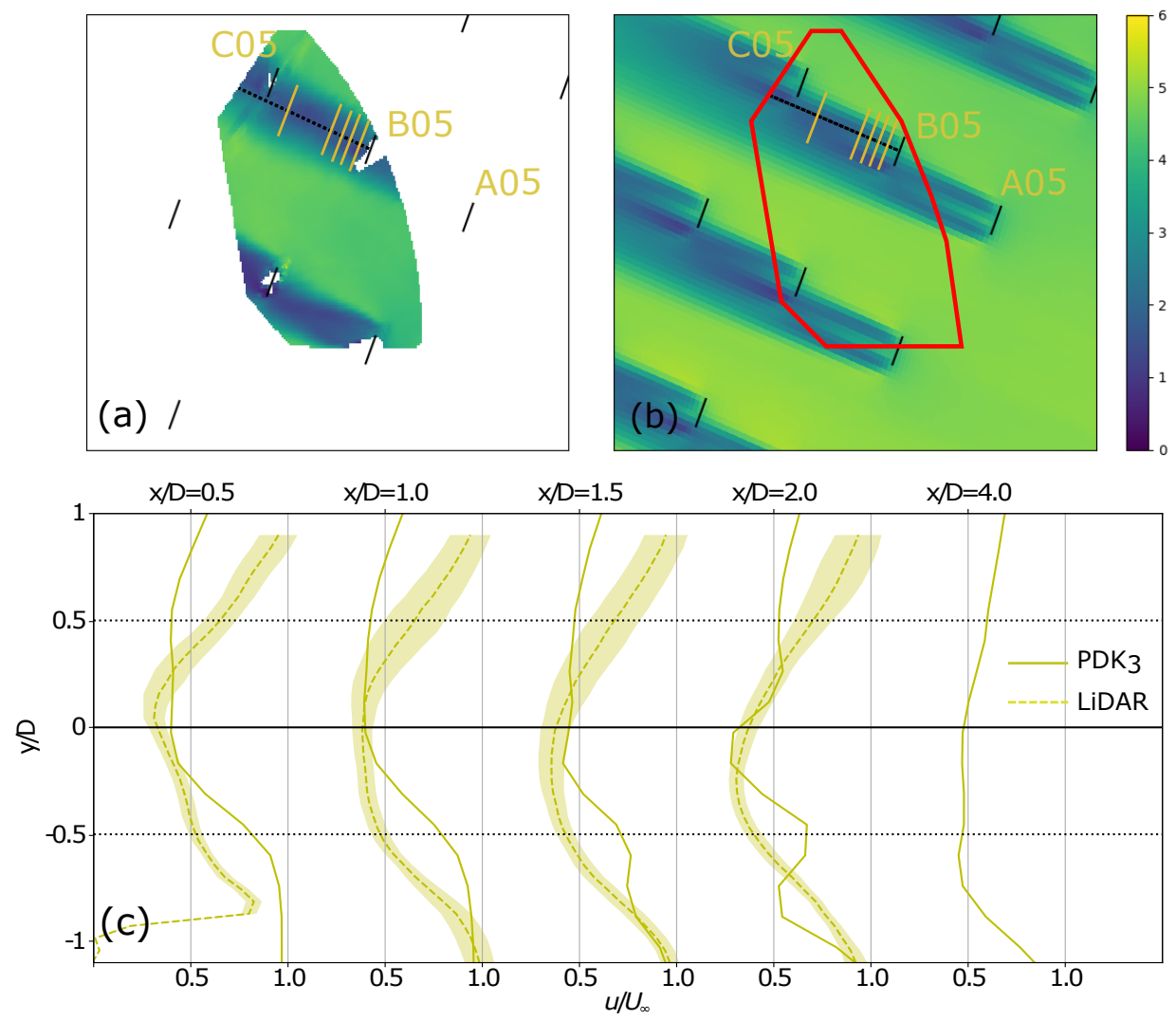

Figure 17. Comparison of LES time averaged velocity (b) against LiDAR wake measurements (a) for case $P D k_{3}$ and turbine B05. (c) Comparison of velocity wake deficit at downstream locations from turbine B05. LiDAR data at $\mathrm{x} / \mathrm{D}=4.0$ was not available.

field, power produced by individual turbines as well as the entire wind-farm, mean and fatigue blade-root loading and individual turbine wakes and recovery. However, limitations of the flow solver were exhibited in certain instances, where higher errors were observed in the loads for turbines operating in waked state, suggesting that the grid resolution in the numerical domain may not be fine enough to capture enough of the relevant smaller turbulent structures in turbine wakes. Additionally, controller mismatch due to lack of information of the field controllers also lead to discrepancies in the produced power. Nevertheless, the results from the validation study are promising, proving the capability of a high-fidelity numerical solver to represent on-field conditions and performance output of a large wind farm.

Future work could include investigating the effect of grid resolution on the simulated results, as well as improving the implemented controller in the simulation environment. This can be achieved by expanding the precursor database with finer resolution data-sets, and a closer co-operation with the turbine manufacturers to obtain a detailed controller description. Having exhibited the capability of the numerical solver in representing normal wind-farm operation, validation studies could also 
be conducted to evaluate the effect of coordinated wind-farm control strategies, such as wake steering and induction control, to improve wind-farm performance.

\section{Appendix A: Multibody model}

\section{A1 Coordinate system}

The rotor of the wind turbine is considered as one single body with each blade modeled using a number of interconnected beam elements, as can be seen in Figure A1. The origin of the body reference $X_{1}^{i} X_{2}^{i} X_{3}^{i}$ is located at the center of the turbine rotor and coincides with the root nodes of the finite element beam representations of the respective blades. The $X_{1}^{i}$ axis is directed along the length of the first blade, while the axis $X_{3}^{i}$ is directed along the axis of rotation of the rotor, in the upwind sense. The location of the origin of the body reference with respect to the global coordinate system $X_{1} X_{2} X_{3}$ (not shown in Fig. 1) is denoted by the vector of Cartesian coordinates $R^{i}$, while the orientation of the body reference w.r.t. the global coordinate system is denoted by the vector of rotational coordinates $\theta^{i}$. Only rotation along the turbine's main axis is assumed to contribute dynamically to the behavior of the turbine; while tilting and yawing motions are taken into account quasi-statically. Finally, the elastic deformations of the rotor are described by the vector of elastic coordinates $q_{f}^{i}$, which describe the displacements of the finite element nodes and the local derivatives thereof. Summarizing, the configuration of the turbine rotor can be described by the following vector of generalized coordinates:

$375 q^{i}=\left[R^{i^{T}} \theta^{i^{T}} q_{f}^{i^{T}}\right]^{T}$

The global position of an arbitrary point on the $j^{\text {th }}$ beam element of the $i^{\text {th }}$ body of the multibody system can be written as

$r^{i j}=R^{i}+A^{i} \bar{u}^{i j}$

where $\overline{\boldsymbol{u}}^{i j}$ is the displacement vector of the $i j^{\text {th }}$ element and $\boldsymbol{A}^{\boldsymbol{i}}$ is the transformation matrix of body $i$, which defines the orientation of the body reference with respect to the global reference. The yaw $(\phi)$, tilt $(\psi)$, rotation $(\theta)$ and precone angles $(\gamma)$ are used as rotational coordinates about their respective axes, and the resulting transformation matrix cased by these rotations is given by

$A^{i}=R_{y}^{-1} R_{t}^{-1} R_{r}^{-1} R_{p}^{-1}$

where $\boldsymbol{R}_{\boldsymbol{y}}, \boldsymbol{R}_{t}, \boldsymbol{R}_{\boldsymbol{r}}$ and $\boldsymbol{R}_{\boldsymbol{p}}$ are the 3-D yaw, tilt, roll and precone rotation matrices.

\section{A2 Energy of the turbine rotor}

The global velocity of a selected point can be determined by differentiating $\boldsymbol{r}^{i j}$ with respect to time to obtain

$\dot{\mathbf{r}}^{i j}=\dot{\mathbf{R}}^{i}+\dot{\mathbf{A}}^{i} \overline{\mathbf{u}}^{i j}+\mathbf{A}^{i} \dot{\mathbf{u}}^{i j}$ 


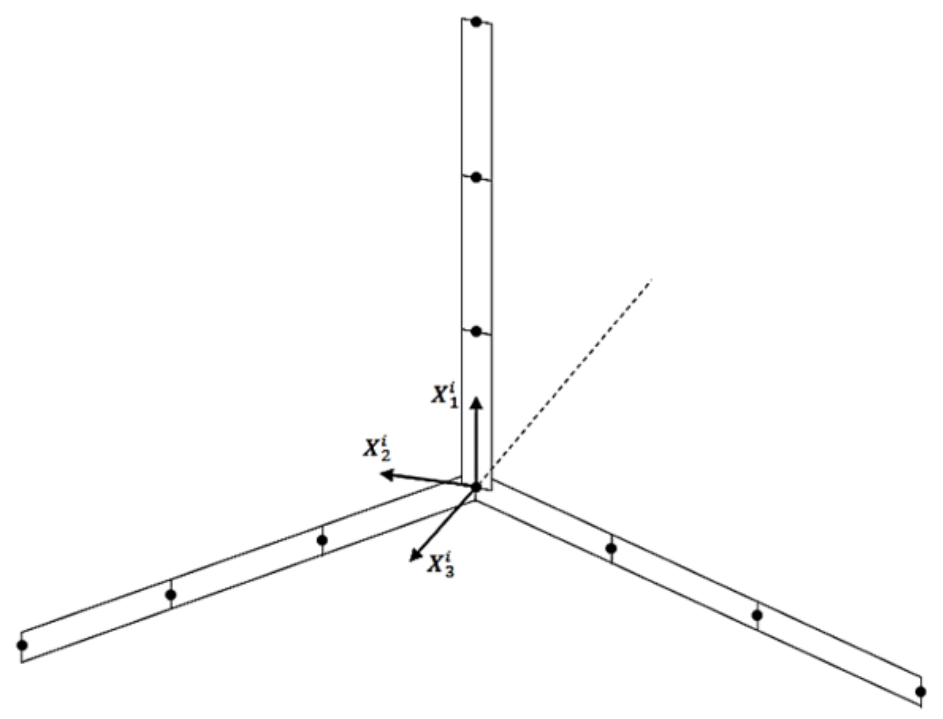

Figure A1. Finite element beam representation of the wind turbine blades.

which can be simplified by taking into account the assumption of quasi-static variation of yaw, tilt and precone $(\dot{\phi}, \dot{\psi}, \dot{\gamma}=0)$. Thus, the kinetic energy $T^{i j}$ of element $i j$ in the finite element representation of the rotor can be obtained by the following formula

$390 \quad T^{i j}=\frac{1}{2} \int_{V^{i j}} \rho^{i j} \mathbf{r}^{i j T} \dot{\mathbf{r}}^{i j} d V^{i j}$

where $V^{i j}$ and $\rho^{i j}$ are the mass density and volume of the $i j^{\text {th }}$ element, respectively. The total kinectic energy of the turbine rotor can be determined by summing up the kinetic energies of all the elements. Using the floating frame of reference approach (Shabana, 2013), the expression for the elastic potential energy takes a very simple form, since only the elastic (i.e. not the rigid body) displacement of the body contributes to the elastic potential energy. Consequently, the potential energy of the $i j^{t h}$ element is given by

$\Pi^{i j}=\frac{1}{2} \mathbf{q}_{f}^{i T} \boldsymbol{K}_{f f}^{i j} \mathbf{q}_{f}^{i}$

where $\boldsymbol{K}_{f f}^{i j}$ is the the element stiffness matrix expressed with respect to the body reference frame.

\section{A3 Equations of motion}

The equations of motion of the turbine rotor are developed from Lagrange's equations for constrained systems. These equations can be written in the following form:

$\frac{d}{d t}\left(\frac{\partial T^{i}}{\partial \dot{\mathbf{q}}^{i}}\right)^{T}-\left(\frac{\partial T^{i}}{\partial \mathbf{q}^{i}}\right)^{T}+\left(\frac{\partial \Pi^{i}}{\partial \mathbf{q}^{i}}\right)^{T}+\boldsymbol{C}_{\boldsymbol{q}^{i}}^{\boldsymbol{T}} \boldsymbol{\lambda}=\mathbf{Q}^{i}$ 
where $T^{i}$ and $\Pi^{i}$ are the kinetic and potential energy of the $i^{t h}$ body, $\mathbf{q}^{i}$ is the vector of generalized coordinates of the $i^{t h}$ body, and $Q^{i}$ is the vector of generalized forces associated with the coordinates of the $i^{\text {th }}$ body. Furthermore, $\lambda$ is the vector of Lagrange multipliers and is the $C_{q}^{i}$ constraint Jacobian matrix, defined as

$\boldsymbol{C}_{\boldsymbol{q}}^{\boldsymbol{i}}=\frac{\partial \mathbf{C}}{\partial \mathbf{q}^{i}}=\left[\begin{array}{cccc}\frac{\partial C_{1}}{\partial q_{1}^{i}} & \frac{\partial C_{1}}{\partial q_{2}^{i}} & \ldots & \frac{\partial C_{1}}{\partial q_{n}^{i}} \\ \frac{\partial C_{2}}{\partial q_{1}^{i}} & \frac{\partial C_{2}}{\partial q_{2}^{i}} & \ldots & \frac{\partial C_{2}}{\partial q_{n}^{i}} \\ \vdots & \vdots & \ddots & \ldots \\ \frac{\partial C_{n_{c}}}{\partial q_{1}^{i}} & \frac{\partial C_{n_{c}}}{\partial q_{2}^{i}} & \ldots & \frac{\partial C_{n_{c}}}{\partial q_{n}^{i}}\end{array}\right]$

where $\mathbf{C}=\mathbf{C}(\mathbf{q}, t)=\left(C_{1} C_{2} \ldots C_{n_{c}}\right)^{T}$ is the vector of linearly independent constraint functions that satisfy the holonomic constraint equations of the multibody system

$\mathbf{C}(\mathbf{q}, t)=0$

After evaluating and expanding the partial derivatives in equation A7 in terms of the mass and stiffness elements of the rotor structure, we can obtain the equation of motion of the multibody structure as

$\mathrm{M}^{\mathrm{i}} \mathrm{q}^{\mathrm{i}}+\mathrm{K}^{\mathrm{i}} \mathrm{q}^{\mathrm{i}}+C_{q}^{T} \lambda=Q_{e}^{i}+Q_{v}^{i}$

where $Q_{e}^{i}$ is the vector of generalized external forces containing the aerodynamic and gravity forces at each body element and $Q_{v}^{i}$ is the quadratic velocity vector of body i, as defined by

$\boldsymbol{Q}_{\boldsymbol{v}}^{i}=\frac{1}{2}\left[\frac{\partial}{\partial \mathbf{q}^{i}}\left(\dot{\mathbf{q}}^{i^{T}} \mathbf{M}^{i} \dot{\mathbf{q}}^{i}\right)\right]^{T}-\dot{\mathbf{M}}^{i} \dot{\mathbf{q}}^{i}$

\section{Appendix B: Coupling of turbine model with flow solver}

During each LES time step, the blades sweep a sector area where the loads and their dynamic response are evaluated in a two-way Fluid-Structure Interaction (FSI) manner. Loads acting on the turbine and tower structure lead to deformations, which are evaluated using equation 3 , and the subsequent loads are then computed on the structure's deformed positions, before being added to the flow equations 1 as body force terms $\overline{\mathbf{F}}$. Before being added to the flow equations, the body forces of are processed by spatial and time filtering, as detailed in the following subsections.

\section{B1 Spatial filter of rotor-swept forces}

First, the unsteady forces $\mathbf{F}(\hat{\mathbf{u}}, \mathbf{q})$ are smeared out in the surrounding LES mesh nodes by taking their convolution with a Gaussian kernel $G_{n}$, resulting in the spatially filtered forces $\hat{F}(x)$ :

$\hat{F}(\mathbf{x})=\sum_{n=1}^{N_{t}} \sum_{j=1}^{N_{b}=3} \int_{0}^{R} F(\hat{u}, q, r) G_{n}\left(\left\|\mathbf{x}-r \mathbf{e}_{\mathbf{j}}\right\|\right) d r$

425 where, $N_{t}$ is the number of turbines, $N_{b}$ the number of blades, and $\left\|\mathbf{x}-r \mathbf{e}_{\mathbf{j}}\right\|$ is the Euclidean distance between the LES grid point and the deflected actuator line point, accounting for structural deformations. 
https://doi.org/10.5194/wes-2021-153

Preprint. Discussion started: 20 January 2022

(c) Author(s) 2022. CC BY 4.0 License.

(c) (i)

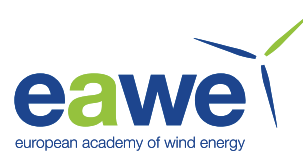

\section{B2 Time filter of rotor-swept forces}

The body forces $\overline{\mathbf{F}}$ of the Navier-Stokes equations are then calculated by time-filtering the Gaussian-filtered forces $\hat{F}$ through a first-order low-pass filter, which gives more weight to the last sub-iterations (see Figure 4d). The time-filter is given as follows:

$$
\frac{d \bar{F}^{i}}{d t}=\frac{1}{\tau_{f}}\left[\hat{F}-\bar{F}^{i}\right]
$$

where, $\bar{F}^{i}$ is the time-filtered force at Runge-Kutta stage $i, \hat{F}$ are the spatially filtered forces through the sub-cycles, and $\tau_{f}$ is the time filter constant. The filter constant $\tau_{f}$ defines the effective sector angle, which is chosen to be equal to the LES time step. Equation B2 is integrated during the sub-iterations of the multibody solver using an implicit Euler scheme.

Author contributions. IS and JM jointly set up the concept and objectives of the current work. IS performed code implementations in the LES code SP-Wind and carried out the wind farm simulations. AV and BB developed and implemented the multibody framework and turbine model. ES conducted the measurement campaign and data analysis under the guidance of GL. IS selected the validation cases with support from JM. IS performed the data analysis and data visualization. IS and JM wrote the manuscript with contributions and revisions from ES, $\mathrm{AV}$ and GL.

Code and data availability. The SP-Wind flow solver is a proprietary software of KU Leuven. The TotalControl inflow database used in this work to recreate the inflow conditions at the Lillgrund wind farm is publicly available on zenodo (Munters et al., 2019a, b, c, d). Raw data of the simulation results can be obtained by contacting the corresponding author.

Competing interests. The authors declare that they have no conflict of interest.

Acknowledgements. The authors have received funding from the European Unions Horizon 2020 program (TotalControl, grant no. 727680).

445 The computational resources and services used in this work were provided by the VSC (Flemish Supercomputer Center), funded by the Research Foundation Flanders (FWO) and the Flemish Government department EWI. The authors also acknowledge DTU, Siemens Gamesa and Vattenfall for providing the LiDAR, loads and SCADA data, respectively. 
https://doi.org/10.5194/wes-2021-153

Preprint. Discussion started: 20 January 2022

(c) Author(s) 2022. CC BY 4.0 License.

\section{References}

Allaerts, D. and Meyers, J.: Large eddy simulation of a large wind-turbine array in a conventionally neutral atmospheric boundary layer,

Physics of Fluids, 27, https://doi.org/10.1063/1.4922339, 2015.

Arnold, M., Brüls, O., Arnold, M., Brüls, O., Arnold, M., and Brüls, O.: Convergence of the generalized- $\alpha$ scheme for constrained mechanical systems To cite this version : HAL Id : hal-01490825 mechanical systems, Multibody System Dynamics, Springer Verlag, 85, 187-202, 2007.

Bastankhah, M. and Porté-Agel, F.: A new miniaturewind turbine for wind tunnel experiments. Part I: Design and performance, Energies, 10, https://doi.org/10.3390/en10070908, 2017.

Boersma, S., Doekemeijer, B. M., Siniscalchi-Minna, S., and van Wingerden, J. W.: A constrained wind farm controller providing secondary frequency regulation: An LES study, Renewable Energy, 134, 639-652, https://doi.org/10.1016/j.renene.2018.11.031, 2019.

Bossanyi, E.: Combining induction control and wake steering for wind farm energy and fatigue loads optimisation, Journal of Physics: Conference Series, 1037, https://doi.org/10.1088/1742-6596/1037/3/032011, 2018.

Bou-Zeid, E., Meneveau, C., and Parlange, M.: A scale-dependent Lagrangian dynamic model for large eddy simulation of complex turbulent flows, Physics of Fluids, 17, 1-18, https://doi.org/10.1063/1.1839152, 2005.

Calaf, M., Meneveau, C., and Meyers, J.: Large eddy simulation study of fully developed wind-turbine array boundary layers, Physics of Fluids, 22, 015 110, https://doi.org/10.1063/1.3291077, 2010.

Castro, I. P.: Rough-wall boundary layers: Mean flow universality, Journal of Fluid Mechanics, 585, 469-485, https://doi.org/10.1017/S0022112007006921, 2007.

Churchfield, M. J., Schreck, S., Martínez-Tossas, L. A., Meneveau, C., and Spalart, P. R.: An advanced actuator line method for wind energy applications and beyond, 35th Wind Energy Symposium, 2017, https://doi.org/10.2514/6.2017-1998, 2017.

Draper, M., Guggeri, A., and Usera, G.: Validation of the Actuator Line Model with coarse resolution in atmospheric sheared and turbulent inflow, Journal of Physics: Conference Series, 753, https://doi.org/10.1088/1742-6596/753/8/082007, 2016.

Frederik, J. A., Doekemeijer, B. M., Mulders, S. P., and van Wingerden, J. W.: The helix approach: Using dynamic individual pitch control to enhance wake mixing in wind farms, Wind Energy, 23, 1739-1751, https://doi.org/10.1002/we.2513, 2020.

Freebury, G. and Musial, W.: Determining equivalent damage loading for full-scale wind turbine blade fatigue tests, 2000 ASME Wind Energy Symposium, pp. 287-297, https://doi.org/10.2514/6.2000-50, 2000.

Göçmen, T. and Giebel, G.: Estimation of turbulence intensity using rotor effective wind speed in Lillgrund and Horns Rev-I offshore wind farms, Renewable Energy, 99, 524-532, https://doi.org/10.1016/j.renene.2016.07.038, 2016.

Goit, J. P. and Meyers, J.: Optimal control of energy extraction in wind-farm boundary layers, Journal of Fluid Mechanics, 768, 5-50, https://doi.org/10.1017/jfm.2015.70, 2015.

Hansen, M. H. and Henriksen, L. C.: Basic DTU Wind Energy controller, January, 2013.

Jiménez, J.: Turbulent flows over rough walls, Annual Review of Fluid Mechanics, 36, 173-196, https://doi.org/10.1146/annurev.fluid.36.050802.122103, 2004.

Kunsch, H. R.: The Jackknife and the Bootstrap for General Stationary Observations, The Annals of Statistics, 17,1217 - 1241 , https://doi.org/10.1214/aos/1176347265, 1989.

Liang, X.: An Integrating Velocity-Azimuth Process Single-Doppler Radar Wind Retrieval Method, Journal of Atmospheric and Oceanic Technology, 24, 658 - 665, https://doi.org/10.1175/JTECH2047.1, 2007. 
https://doi.org/10.5194/wes-2021-153

Preprint. Discussion started: 20 January 2022

(c) Author(s) 2022. CC BY 4.0 License.

(c) (i)

Lin, M. and Porté-Agel, F.: Large-eddy simulation of yawedwind-turbine wakes: comparisons withwind tunnel measurements and analyticalwake models, Energies, 12, 1-18, https://doi.org/10.3390/en12234574, 2019.

Mehta, D., van Zuijlen, A. H., Koren, B., Holierhoek, J. G., and Bijl, H.: Large Eddy Simulation of wind farm aerodynamics: A review, Journal of Wind Engineering and Industrial Aerodynamics, 133, 1-17, https://doi.org/10.1016/j.jweia.2014.07.002, 2014.

Munters, W. and Meyers, J.: Dynamic strategies for yaw and induction control of wind farms based on large-eddy simulation and optimization, Energies, 11, https://doi.org/10.3390/en11010177, 2018.

Munters, W., Meneveau, C., and Meyers, J.: Turbulent Inflow Precursor Method with Time-Varying Direction for Large-Eddy Simulations and Applications to Wind Farms, Boundary-Layer Meteorology, 159, 305-328, https://doi.org/10.1007/s10546-016-0127-z, 2016.

Munters, W., Sood, I., and Meyers, J.: Precursor dataset PDk, https://doi.org/10.5281/zenodo.2650100, 2019a.

Munters, W., Sood, I., and Meyers, J.: Precursor dataset PDkhi, https://doi.org/10.5281/zenodo.2650102, 2019b.

Munters, W., Sood, I., and Meyers, J.: Precursor dataset CNk2, https://doi.org/10.5281/zenodo.2650096, 2019c.

Munters, W., Sood, I., and Meyers, J.: Precursor dataset CNk4, https://doi.org/10.5281/zenodo.2650098, 2019d.

Nilsson, K., Ivanell, S., Hansen, K. S., Mikkelsen, R., Sørensen, J. N., Breton, S.-P., and Henningson, D.: Large-eddy simulations of the Lillgrund wind farm, Wind Energy, pp. 449-467, https://doi.org/10.1002/we.1707, 2014.

Sebastiani, A., Castellani, F., Crasto, G., and Segalini, A.: Data analysis and simulation of the Lillgrund wind farm, Wind Energy, 24, 634-648, https://doi.org/https://doi.org/10.1002/we.2594, 2021.

Shabana, A. A.: Dynamics of Multibody Systems, Cambridge University Press, 4 edn., https://doi.org/10.1017/CBO9781107337213, 2013.

Simisiroglou, N., Polatidis, H., and Ivanell, S.: Wind farm power production assessment: a comparative analysis of two actuator disc methods and two analytical wake models, Wind farm power production assessment: a comparative analysis of two actuator disc methods and two analytical wake models, 2018, 1-13, https://doi.org/10.5194/wes-2018-8, 2018.

Simon, E. and Courtney, M.: A Comparison of sector-scan and dual Doppler wind measurements at Høvsøre Test Station - one lidar or two?, 2016.

Socie, D. and Downing, S.: Simple Rainflow Counting Algorithms, International Journal of Fatigue, 4, 31-40, http://masters.donntu.org/ 2015/fimm/zinchencko/library/article11.pdf, 1982.

Sood, I., Meyers, J., and Lanzilao, L.: TotalControl D 1.8 Coupling of Gaussian wake merging to background ABL model, 2020.

510 Stevens, R. J., Graham, J., and Meneveau, C.: A concurrent precursor inflow method for Large Eddy Simulations and applications to finite length wind farms, Renewable Energy, 68, 46-50, https://doi.org/10.1016/j.renene.2014.01.024, 2014.

Storey, R. C., Norris, S. E., and Cater, J. E.: An actuator sector method for efficient transient wind turbine simulation, Wind Energy, 18, 699-711, https://doi.org/10.1002/we.1722, 2015.

Sutherland: Fatigue analysis of wind turbines. Technical report, Sandia National Laboratories, Tech. rep., 1999.

Townsend: The Structure of Turbulent Shear Flow. Cambridge University Press, 1976.

Vasiljević, N., Lea, G., Courtney, M., Cariou, J.-P., Mann, J., and Mikkelsen, T.: Long-Range WindScanner System, Remote Sensing, 8, https://doi.org/10.3390/rs8110896, 2016.

Virtanen, P., Gommers, R., Oliphant, T. E., Haberland, M., Reddy, T., Cournapeau, D., Burovski, E., Peterson, P., Weckesser, W., Bright, J., van der Walt, S. J., Brett, M., Wilson, J., Millman, K. J., Mayorov, N., Nelson, A. R. J., Jones, E., Kern, R., Larson, E., Carey, C. J., Polat, İ., Feng, Y., Moore, E. W., VanderPlas, J., Laxalde, D., Perktold, J., Cimrman, R., Henriksen, I., Quintero, E. A., Harris, C. R., Archibald, A. M., Ribeiro, A. H., Pedregosa, F., van Mulbregt, P., and SciPy 1.0 Contributors: SciPy 1.0: Fundamental Algorithms for Scientific Computing in Python, Nature Methods, 17, 261-272, https://doi.org/10.1038/s41592-019-0686-2, 2020. 
https://doi.org/10.5194/wes-2021-153

Preprint. Discussion started: 20 January 2022

(C) Author(s) 2022. CC BY 4.0 License.

(c) (1)

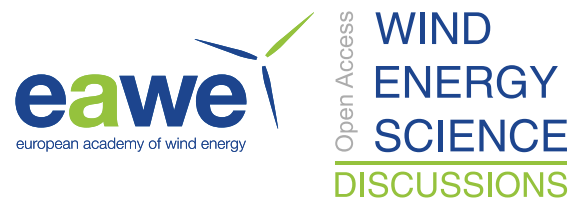

Vitsas, A. and Meyers, J.: Multiscale aeroelastic simulations of large wind farms in the atmospheric boundary layer, Journal of Physics:

Conference Series, 753, 082 020, https://doi.org/10.1088/1742-6596/753/8/082020, 2016.

525 Wu, Y. T. and Porté-Agel, F.: Large-Eddy Simulation of Wind-Turbine Wakes: Evaluation of Turbine Parametrisations, Boundary-Layer Meteorology, 138, 345-366, https://doi.org/10.1007/s10546-010-9569-x, 2011.

Wu, Y. T. and Porté-Agel, F.: Simulation of Turbulent Flow Inside and Above Wind Farms: Model Validation and Layout Effects, BoundaryLayer Meteorology, 146, 181-205, https://doi.org/10.1007/s10546-012-9757-y, 2013.

Wu, Y. T. and Porté-Agel, F.: Modeling turbine wakes and power losses within a wind farm using LES: An application to the Horns Rev offshore wind farm, Renewable Energy, 75, 945-955, https://doi.org/10.1016/j.renene.2014.06.019, 2015.

Y1lmaz, A. E. and Meyers, J.: Optimal dynamic induction control of a pair of inline wind turbines, Physics of Fluids, 30, https://doi.org/10.1063/1.5038600, 2018. 